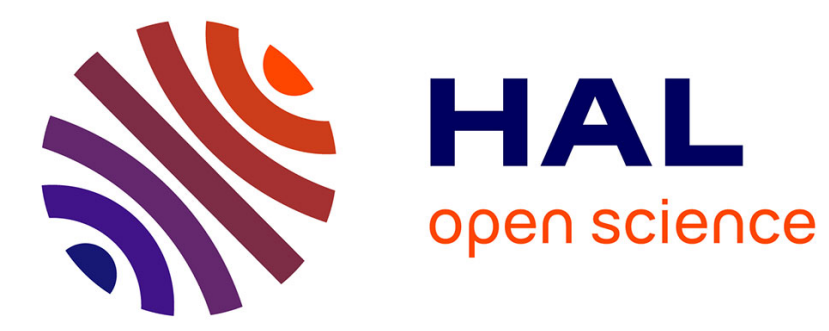

\title{
Experiments on growth series of braid groups
}

\author{
Jean Fromentin
}

\section{To cite this version:}

Jean Fromentin. Experiments on growth series of braid groups. 2021. hal-02929264v3

\section{HAL Id: hal-02929264 \\ https://hal.science/hal-02929264v3}

Preprint submitted on 14 Apr 2021

HAL is a multi-disciplinary open access archive for the deposit and dissemination of scientific research documents, whether they are published or not. The documents may come from teaching and research institutions in France or abroad, or from public or private research centers.
L'archive ouverte pluridisciplinaire $\mathbf{H A L}$, est destinée au dépôt et à la diffusion de documents scientifiques de niveau recherche, publiés ou non, émanant des établissements d'enseignement et de recherche français ou étrangers, des laboratoires publics ou privés. 


\title{
EXPERIMENTS ON GROWTH SERIES OF BRAID GROUPS
}

\author{
JEAN FROMENTIN \\ In memory of Patrick Dehornoy, a great mentor.
}

\begin{abstract}
We introduce an algorithmic framework to investigate spherical and geodesic growth series of braid groups relatively to the Artin's or BirmanKo-Lee's generators. We present our experimentations in the case of three and four strands and conjecture rational expressions for the spherical growth series with respect to the Birman-Ko-Lee's generators.
\end{abstract}

\section{INTRODUCTION}

Originally introduced as the group of isotopy classes of $n$-strands geometric braids, the braid group $B_{n}$ admits many finite presentations by generators and relations. From each finite semigroup generating set $S$ of $B_{n}$ we can define at least two growth series. The spherical growth series counts elements of $B_{n}$ by their distance from the identity in the Cayley graph Cay $\left(B_{n}, S\right)$ of $B_{n}$ with respect to $S$. The geodesic growth series counts geodesic paths starting from the identity by length in $\operatorname{Cay}\left(B_{n}, S\right)$.

In case of Artin's generators $\Sigma_{n}=\left\{\sigma_{1}^{ \pm 1}, \ldots, \sigma_{n-1}^{ \pm 1}\right\}$ of $B_{n}$ the only known significant results are for $n \leqslant 3$. L. Sabalka determines [24] both the spherical and geodesic growth series of $B_{3}$. To this end, he constructs an explicit deterministic finite automaton recognizing the language of geodesic $\Sigma_{3}$-words. In particular he obtains the rationality of both series. Similar results were obtained by J. Mairesse and F. Mathéus in case of Artin-Tits groups of dihedral type [23]. In page 57 of her $\mathrm{PhD}$ thesis [2], M. Albenque gives the first 13 terms of the spherical series of $B_{4}$ relatively to $\Sigma_{4}$.

Here we introduce a new algorithmic framework to compute the first terms of the spherical and geodesic growth series of $B_{n}$ relatively to both Artin's or BirmanKo-Lee's generators. Experimentations allow us to conjecture rational expressions for the spherical growth series of $B_{3}$ and $B_{4}$ and geodesic growth series of $B_{3}$ relatively to the Birman-Ko-Lee's generators. We also obtain the first 26 terms of the spherical and geodesic growth series of $B_{4}$ with respect to $\Sigma_{4}$ but this is not enough to formulate any conjecture in this case. Experiments presented in this paper were carried out using the CALCULCO computing platform [25].

The paper is organized as follows. Section 2 recalls basic definitions and presents already known result on the subject. In section 3 we describe a first algorithm to explore spherical and geodesic combinatorics of braids relatively to Artin's or Birman-Ko-Lee generators. Section 4 is devoted to the notion of braid template which allows us to parallelize the algorithms obtained in the previous section. In

2020 Mathematics Subject Classification. Primary 20F36, 20F10; Secondary 20F69, 68R15.

Key words and phrases. Braid group, spherical growth series, geodesic growth series, algorithm. 
section 5 we show how to reduce the exploration space by introducing reduced braid templates. Experimentation results are detailed in the last section.

\section{Context}

2.1. Growth series. Let $S$ be a finite generating set of a semigroup $M$. We denote by $S^{*}$ the set of all words on the alphabet $S$, which are called $S$-words. The empty word is denoted by $\varepsilon$. For every $S$-word $u$, we denote by $|u|$ its length and by $\bar{u}$ the element of $M$ it represents. We say that two $S$-words $u$ and $v$ are equivalent, denoted $u \equiv v$, is they represent the same element in $M$.

Definition 2.1. The $S$-length of an element $x \in M$, denoted $|x|_{S}$, is the length of a shortest $S$-word representing $x$. An $S$-word $u$ satisfying $|u|=|\bar{u}|_{S}$ is geodesic.

The $S$-length of an element $x \in M$ corresponds to the distance between $x$ and the identity in the Cayley graph of $M$ with respect to the finite generating set $S$.

Definition 2.2. For any $\ell \in \mathbb{N}$, we denote by $g(M, S ; \ell)$ the number of geodesic $S$-words of length $\ell$. The geodesic growth series of $M$ with respect to $S$ is

$$
\mathcal{G}(M, S)=\sum_{\ell \in \mathbb{N}} g(M, S ; \ell) t^{\ell}
$$

If the language of geodesic $S$-words is regular then the series $\mathcal{G}(M, S)$ is rational.

Definition 2.3. For any $\ell \in \mathbb{N}$, we denote by $s(M, S ; \ell)$ the number of elements in $M$ of length $\ell$. The spherical growth series of $M$ with respect to $S$ is

$$
\mathcal{S}(M, S)=\sum_{x \in M} t^{|x|_{S}}=\sum_{\ell \in \mathbb{N}} s(M, S ; \ell) t^{\ell} .
$$

If there exists a regular language composed of geodesic $S$-words in bijection with $M$ then the series $\mathcal{S}(M, S)$ is rational.

2.2. Artin's braid presentation. The first presentation of the braid group $B_{n}$ was given by E. Artin in [4] :

$$
B_{n} \simeq\left\langle\sigma_{1}, \ldots, \sigma_{n-1} \mid \begin{array}{cc}
\sigma_{i} \sigma_{j}=\sigma_{j} \sigma_{i} & \text { for }|i-j| \geqslant 2 \\
\sigma_{i} \sigma_{j} \sigma_{i}=\sigma_{j} \sigma_{i} \sigma_{j} & \text { for }|i-j|=1
\end{array}\right\rangle .
$$

Definition 2.4. For all $n \geqslant 2$, we denote by $\Sigma_{n}^{+}$the set $\left\{\sigma_{1}, \ldots, \sigma_{n-1}\right\}$ and by $\Sigma_{n}$ the set $\Sigma_{n}^{+} \sqcup\left(\Sigma_{n}^{+}\right)^{-1}$.

Artin's presentation of $B_{n}$ implies that $\Sigma_{n}^{+}$is a set of group generators of $B_{n}$. However the braid $\sigma_{1}^{-1}$ cannot be represented by any $\Sigma_{n}^{+}$-word. For our purpose, it is fundamental to view a monoid (or a group) as a quotient of a finitely generated free monoid. As a monoid, the braid group $B_{n}$ is presented by generators $\Sigma_{n}$ and the relations of (1) plus relations

$$
\sigma_{i} \sigma_{i}^{-1}=\sigma_{i}^{-1} \sigma_{i}=\varepsilon \quad \text { for all } 1 \leqslant i \leqslant n-1 .
$$

In [24], L. Sabalka constructed an explicit deterministic finite state automaton recognizing the language of geodesic $\Sigma_{3}$-words. He obtained the following rational value for the geodesic growth series of $B_{3}$ relatively to the Artin's generators $\Sigma_{3}$ :

$$
\mathcal{G}\left(B_{3}, \Sigma_{3}\right)=\frac{t^{4}+3 t^{3}+t+1}{\left(t^{2}+2 t-1\right)\left(t^{2}+t-1\right)} .
$$



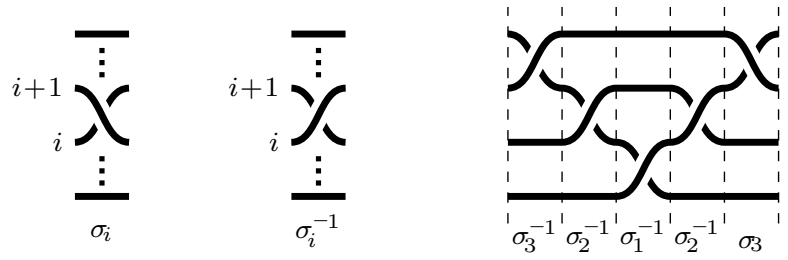

FiguRE 1. Geometric interpretation of Artin's generators and representation of a 4 -strands braid as a $\Sigma_{4}$-word.

Moreover, using the finite state automaton recognizing the language of short-lex normal form of $B_{3}[17]$ he obtains :

$$
\mathcal{S}\left(B_{3}, \Sigma_{3}\right)=\frac{(t+1)\left(2 t^{3}-t^{2}+t-1\right)}{(t-1)(2 t-1)\left(t^{2}+t-1\right)} .
$$

The positive braid monoid $B_{n}^{+}$is the submonoid of $B_{n}$ generated by $\Sigma_{n}^{+}$. Since every $\Sigma_{n}^{+}$-word is geodesic, the geodesic growth series $\mathcal{G}\left(B_{n}^{+}, \Sigma_{n}^{+}\right)$is irrelevant. An explicit rational formula for the spherical growth series $\mathcal{S}\left(B_{n}^{+}, \Sigma_{n}^{+}\right)$was obtained by A. Bronfman in [8] and later by M. Albenque in [1]. These results were extended to positive braid monoids of type B and D in [3] and for each Artin-Tits monoids of spherical type in [18].

2.3. Dual's braid presentation. In [7], J. Birman, K. H. Ko and S. J. Lee introduced a new generator family of $B_{n}$, called Birman-Ko-Lee's or dual generators.

Definition 2.5. For $1 \leqslant p<q \leqslant n$ we define $a_{p, q}$ to be the braid

$$
a_{p, q}=\sigma_{p} \ldots \sigma_{q-2} \sigma_{q-1} \sigma_{q-2}^{-1} \ldots \sigma_{p}^{-1} .
$$

For all $n \geqslant 2$, we put $\Sigma_{n}^{+*}=\left\{a_{p, q} \mid 1 \leqslant p<q \leqslant n\right\}$ and $\Sigma_{n}^{*}=\Sigma_{n}^{+*} \sqcup\left(\Sigma_{n}^{+*}\right)^{-1}$.

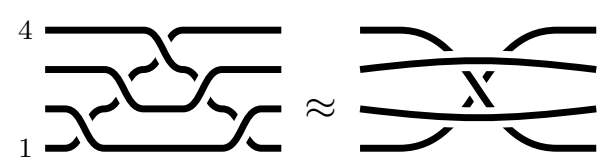

Figure 2. The letter $a_{1,4}$ codes for the braid in which strands 1 and 4 cross under strands 2 and 3 .

We write $[p, q]$ for the interval $\{p, \ldots, q\}$ of $\mathbb{N}$, and we say that $[p, q]$ is nested in $[r, s]$ if we have $r<p<q<s$.

Lemma 2.6. [7] In terms of $\Sigma_{n}^{+*}$, the group $B_{n}$ is presented by the relations

$$
\begin{aligned}
& a_{p, q} a_{r, s}=a_{r, s} a_{p, q} \quad \text { for }[p, q] \text { and }[r, s] \text { disjoint or nested, } \\
& a_{p, q} a_{q, r}=a_{q, r} a_{p, r}=a_{p, r} a_{p, q} \quad \text { for } 1 \leq p<q<r \leq n .
\end{aligned}
$$

Note that the definition of $a_{p, q}$ given here is not exactly that of [7] but it is coherent with previous papers of the author.

As for Artin's generators, the braid group $B_{n}$ admits a monoid presentation with generators $\Sigma_{n}^{*}$, relations (6) and (7) together with

$$
a_{p, q} a_{p, q}^{-1}=a_{p, q}^{-1} a_{p, q}=\varepsilon \quad \text { for all } 1 \leqslant p<q \leqslant n .
$$


Except in the case $n=2$, which is trivial, there are no results in the literature on the growth series of $B_{n}$ with respect to $\Sigma_{n}^{*}$.

The Birman-Ko-Lee monoid $B_{n}^{+*}$, also called dual braid monoid in [5] is the submonoid of $B_{n}$ generated by $\Sigma_{n}^{+*}$. The term dual was used by D. Bessis since the Garside structure of $B_{n}^{+}$and $B_{n}^{+*}$ share symmetric combinatorial values. In [3], M. Albenque and P. Nadeau give a rational expression for the spherical growth series $\mathcal{S}\left(B_{n}^{+*}, \Sigma_{n}^{+*}\right)$; they also treat the case of dual braid monoids of type $\mathbf{B}$.

2.4. Some words about Garside presentations. The two monoids $B_{n}^{+}$and $B_{n}^{+*}$ equip the braid group $B_{n}$ with two Garside structures : the classical one [21] and the dual one $[7,5]$. The reader can consult [14] and [13] for a general introduction to Garside theory. Here it is sufficient to know that each Garside structure provides simple elements which generate the corresponding Garside monoid. Let us denote by $C_{n}$ and $D_{n}$ the simple elements of the Garisde monoid $B_{n}^{+}$and $B_{n}^{+*}$ respectively.

In [11], P. Dehornoy starts the study of the spherical combinatorics of $B_{n}^{+}$relatively to $C_{n}$. In particular he formulates a divisibility conjecture which has been proven by F. Hivert, J.-C. Novelli and J.-Y. Thibon in [22]. A similar result was obtained for braid monoids of type $\mathbf{B}$ in [19]. The spherical combinatorics of $B_{n}^{+*}$ relatively to $D_{n}$ was also considered by P. Biane and P. Dehornoy in [6]: they reduce the computation of $s\left(B_{n}^{+*}, D_{n} ; 2\right)$ to that of free cumulants for a product of independent variables.

R. Charney establishes in [9] that the spherical growth series of Artin-Tits groups of spherical type with respect to their standard simple elements are rationals. In particular she obtains the rationality of $\mathcal{S}\left(B_{n}, C_{n}\right)$. This result was generalized for all Garside groups by P. Dehornoy in [10]. This implies in particular the rationality of $\mathcal{S}\left(B_{n}, D_{n}\right)$.

\section{Counting BRAids}

We fix an integer $n \geqslant 2$ and $S_{n}$ denotes either $\Sigma_{n}$ (Artin's generators of $B_{n}$ ) or $\Sigma_{n}^{*}$ (dual generators of $B_{n}$ ).

Definition 3.1. For $n \geqslant 2$ and $\ell \in \mathbb{N}$ we denote by $B_{n}\left(S_{n}, \ell\right)$ the set of braids of $B_{n}$ whose $S_{n}$-length is $\ell$.

Since the equality $s\left(B_{n}, S_{n} ; \ell\right)=\operatorname{card}\left(B_{n}\left(S_{n}, \ell\right)\right)$ holds, we compute $s\left(B_{n}, S_{n} ; \ell\right)$ by constructing the set $B_{n}\left(S_{n}, \ell\right)$. Each braid of $B_{n}$ with $S_{n}$-length $\ell$ is the product of a braid of $S_{n}$-length $\ell-1$ and a generator $x \in S_{n}$. In particular we have

$$
B_{n}\left(S_{n}, \ell\right) \subseteq\left\{\beta \cdot x \text { for }(\beta, x) \in B_{n}\left(S_{n}, \ell-1\right) \times S_{n}\right\},
$$

and so we can construct $B_{n}\left(S_{n}, \ell\right)$ by induction on $\ell \geqslant 1$.

3.1. Representative sets. From an algorithmic point of view, a braid is naturally represented by a word. We extend this notion to any subset of $B_{n}\left(S_{n}, \ell\right)$.

Definition 3.2. We say that a set $W$ of $S_{n}$-words represents a subset $X$ of $B_{n}$ whenever $W$ is a set of unique geodesic representatives for $X$.

Example 3.3. For all $n \geqslant 2$, the set $\{\varepsilon\}$ represents $B_{n}\left(S_{n}, 0\right)$. Since relations (1), (2), together with relations (6)-(8) of Artin and dual semigroup presentation of $B_{n}$ preserve parity of word length we have the following property:

two $S_{n}$-words $u$ and $v$ are equivalent only if $|u| \equiv|v| \bmod 2$. 
In particular any $S_{n}$-word of length $\leqslant 1$ is geodesic. As two different letters of $S_{n}$ represent different braids of $B_{n}$ the set $S_{n}$ represents $B_{n}\left(S_{n}, 1\right)$.

The previous example gives a representative set of $B_{n}\left(S_{n}, \ell\right)$ for $\ell \leqslant 1$. We now tackle the construction of a representative set $W_{\ell}$ of $B_{n}\left(S_{n}, \ell\right)$ for $\ell \geqslant 2$. Using an inductive argument we can assume we already have obtained a set $W_{\ell-1}$ representing $B_{n}\left(S_{n}, \ell-1\right)$ and then consider the set

$$
W^{\prime}=\left\{w x \text { for }(w, x) \in W_{\ell-1} \times S_{n}\right\} .
$$

A first step to obtain $W_{\ell}$ consists in removing all non-geodesic words from $W^{\prime}$. For this we have to test if a given word of $W^{\prime}$ is geodesic or not. A naive general solution consists in testing if a word $u \in W^{\prime}$ is equivalent to a $S_{n}$-word of length at most $\ell-1$. However, as words of $W^{\prime}$ are obtained by appending a letter to a geodesic word, we can restrict the search space:

Lemma 3.4. For $\ell \geqslant 2$, let $u$ be a geodesic $S_{n}$-word of length $\ell-1$ and $x$ a letter of $S_{n}$. If the $S_{n}$-word $v=u x$ is not geodesic then there exists a geodesic $S_{n}$-word $w$ of length $\ell-2$ which is equivalent to $v$.

Proof. Assume $v$ is not geodesic. There exists a $S_{n}$-geodesic word $w$ equivalent to $v$ and satisfying $|w|<|v|$. By (10) we must have $|w| \leqslant|v|-2=\ell-2$. From the equality $v=u x$ we obtain $u \equiv v x^{-1}$ and so $u \equiv w x^{-1}$. Since $u$ is geodesic we must have $\left|w x^{-1}\right| \geqslant \ell-1$, implying $|w| \geqslant \ell-2$ and so $|w|=\ell-2$.

3.2. Geodesic words. For all $\ell \in \mathbb{N}$ the number $g\left(B_{n}, S_{n} ; \ell\right)$ can be obtained at no cost during the construction of a representative set of $B_{n}\left(S_{n}, \ell\right)$.

Definition 3.5. For a braid $\beta \in B_{n}$ we denote by $\omega_{S_{n}}(\beta)$ the number of geodesic $S_{n}$-words representing $\beta$.

Proposition 3.6. For $\beta \in B_{n}$ a braid with $\ell=|\beta|_{S_{n}} \geqslant 1$, we have

$$
\omega_{S_{n}}(\beta)=\sum_{\substack{x \in S_{n} \\\left|\beta x^{-1}\right| S_{n}=\ell-1}} \omega_{S_{n}}\left(\beta x^{-1}\right) .
$$

Proof. Let $W$ be the set of geodesic $S_{n}$-words representing $\beta$. The cardinality of $W$ is then $\omega_{S_{n}}(\beta)$. For all $x \in S_{n}$ we denote by $W_{x}$ the words of $W$ ending with $x$. Since all words of $W$ have length $\ell \geqslant 1$ we must have

$$
W=\bigsqcup_{x \in S_{n}} W_{x} .
$$

Let us fix an element $y \in S_{n}$. By construction, any word of $W_{y}$ has length $\ell-1$, represents the braid $\beta y^{-1}$ and is geodesic. Hence $W_{y}$ is not empty if and only if the $S_{n}$-length of $\beta y^{-1}$ is $\ell-1$, which gives

$$
\omega_{S_{n}}(\beta)=\operatorname{card}(W)=\sum_{x \in S_{n}} \operatorname{card}\left(W_{x}\right)=\sum_{\substack{x \in S_{n} \\\left|\beta x^{-1}\right| S_{n}=\ell-1}} \operatorname{card}\left(W_{x}\right) .
$$

Assume $\beta y^{-1}$ has $S_{n}$-length $\ell-1$. Since for any geodesic $S_{n}$-word $v$ representing $\beta y^{-1}$, the word $v y$ is a geodesic representative of $\beta$, the braid $\beta y^{-1}$ has exactly $\omega_{S_{n}}\left(\beta y^{-1}\right)$ geodesic representatives in $W_{y}$. Therefore card $\left(W_{y}\right)$ is $\omega_{S_{n}}\left(\beta y^{-1}\right)$ and the result follows. 
3.3. A first algorithm. We can now give a first algorithm returning a representative set $W_{\ell}$ of $B_{n}\left(S_{n}, \ell\right)$ for $\ell \geqslant 2$. In order to determine $g\left(B_{n}, S_{n} ; \ell\right)$ we also compute the value of $\omega_{S_{n}}$ for all words in $W_{\ell}$.

In order to construct by induction a representative set $W$, we must test if a given word $u$ is equivalent to a word occuring in $W$ :

Definition 3.7. For a set $W$ of $S_{n}$-words we say that a $S_{n}$-word $u$ appears in $W$, denoted by $u \triangleleft W$, if $u$ is equivalent to a word $v$ of $W$.

In an algorithmic context a $S_{n}$-word is represented as an array of integers plus another integer $\omega$ which eventually correspond to $\omega_{S_{n}}(\bar{u})$. Whenever two variables $\mathrm{u}$ and v stand for the $S_{n}$-words $u$ and $v$ we use:

$-\mathrm{u} \cdot \omega$ to design the integer $\omega$ associated to the word $u$;

$-\mathrm{u} v$ to design the product $u v$.

Algorithm $1-$ REPSET : For $\ell \geqslant 2$, returns a set $\mathrm{W}_{\ell}$ representing $B_{n}\left(S_{n}, \ell\right)$ from two sets $\mathrm{W}_{\ell-1}$ and $\mathrm{W}_{\ell-2}$ representing $B_{n}\left(S_{n}, \ell-1\right)$ and $B_{n}\left(S_{n}, \ell-2\right)$ respectively. For each word $u \in \mathrm{W}_{\ell-1}$, the value of $\mathrm{u} \cdot \omega$ is assumed to be $\omega_{S_{n}}(\bar{u})$.

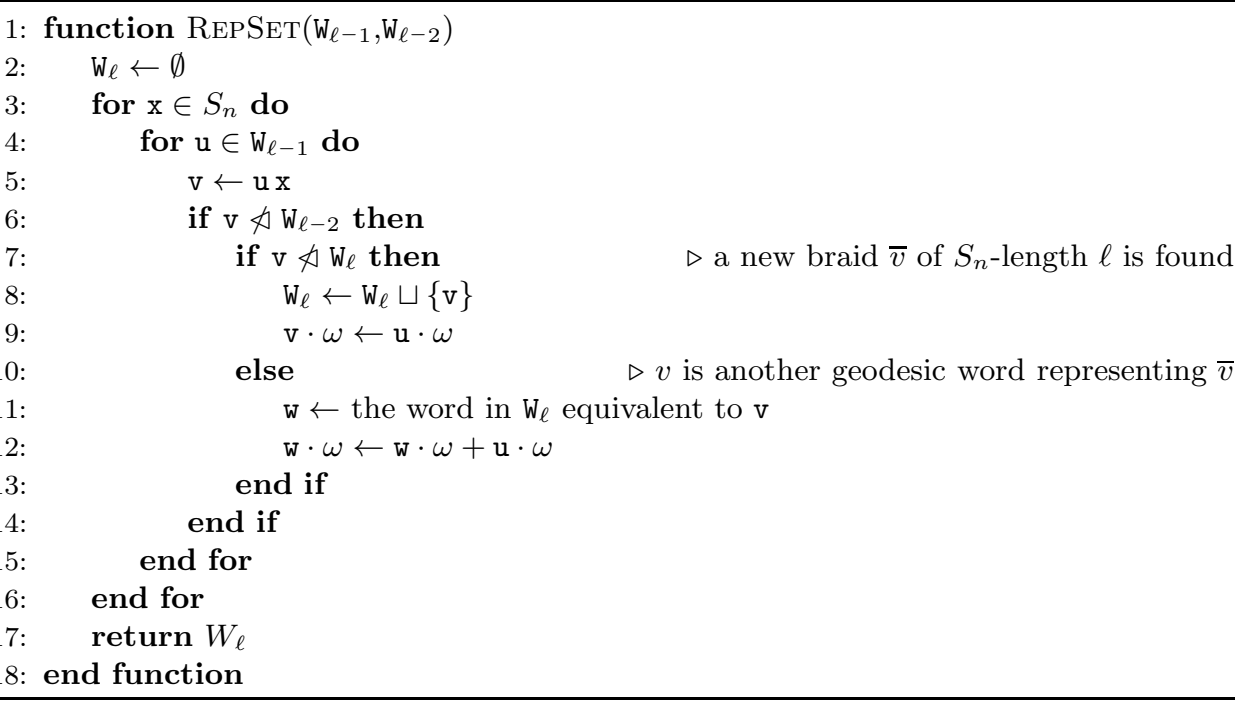

Proposition 3.8. Let $\ell \geqslant 2$ be an integer. Running on sets $W_{\ell-1}$ and $W_{\ell-2}$ representing $B_{n}\left(S_{n}, \ell-1\right)$ and $B_{n}\left(S_{n}, \ell-2\right)$ respectively, algorithm REPSET returns a representing set $W_{\ell}$ of $B_{n}\left(S_{n}, \ell\right)$. Moreover for all $u \in W_{\ell}$, the value of $\mathrm{u} \cdot \omega$ is the integer $\omega_{S_{n}}(\bar{u})$.

Proof. Let $W^{\prime}$ be the set of (11) and $W_{\ell}$ be the set returning by RePSet. The two "for loops" on line 3 and 4 guarantee $W_{\ell} \subseteq W^{\prime}$. By lemma 3.4 and hypotheses on $W_{\ell-1}$ and $W_{\ell-2}$, condition $v \pitchfork W_{\ell-2}$ of line 6 tests if the word $v=u x$ is geodesic. The second if statement line 7 guarantees we append a word $v$ in $W_{\ell}$ if and only if $v$ does not appear in $W_{\ell}$. The set $W_{\ell}$ is then a representative set of $B_{n}\left(S_{n}, \ell\right)$. The result about $\omega_{S_{n}}$ is a direct consequence of Proposition 3.6.

To be complete we must explain how to test if a $S_{n}$-word $u$ appears in a set of $S_{n}$-words. This can be achieved using a normal form (like the Garside's normal form) but such a normal form doesn't provide geodesic representatives. As, for our 
future research, we want to store braids using geodesic representatives, we prefer to use another method.

3.4. Dynnikov's coordinates. Originally defined in [16] from the geometric interpretation of the braid group $B_{n}$ as the mapping class group of the $n$-punctured disk of $\mathbb{R}^{2}$, the Dynnikov's coordinates admit a purely algebraic definition from the action of $B_{n}$ on $\mathbb{Z}^{2 n}$.

For $x \in \mathbb{Z}$, we denote by $x^{+}$the non-negative integer $\max (x, 0)$ and by $x^{-}$the non-positive integer $\min (x, 0)$. We first define an action of Artin's generators on $\mathbb{Z}^{4}$.

Definition 3.9. For all $i \in[1, n-1]$ and all $\left(x_{1}, y_{1}, x_{2}, y_{2}\right) \in \mathbb{Z}^{4}$ we put

$$
\left(x_{1}, y_{1}, x_{2}, y_{2}\right) \cdot \sigma_{i}=\left(x_{1}^{\prime}, y_{1}^{\prime}, x_{2}^{\prime}, y_{2}^{\prime}\right) \quad \text { and } \quad\left(x_{1}, y_{1}, x_{2}, y_{2}\right) \cdot \sigma_{i}^{-1}=\left(x_{1}^{\prime \prime}, y_{1}^{\prime \prime}, x_{2}^{\prime \prime}, y_{2}^{\prime \prime}\right)
$$

where

$$
\begin{array}{ll}
x_{1}^{\prime}=x_{1}+y_{1}^{+}+\left(y_{2}^{+}-t_{1}\right)^{+} & x_{1}^{\prime \prime}=x_{1}-y_{1}^{+}-\left(y_{2}^{+}+t_{2}\right)^{+} \\
y_{1}^{\prime}=y_{2}-t_{1}^{+} & y_{1}^{\prime \prime}=y_{2}+t_{2}^{-} \\
x_{2}^{\prime}=x_{2}+y_{2}^{-}+\left(y_{1}^{-}+t_{1}\right)^{-} & x_{2}^{\prime \prime}=x_{2}-y_{2}^{-}-\left(y_{1}^{-}-t_{2}\right)^{-} \\
y_{2}^{\prime}=y_{1}+t_{1}^{+} & y_{2}^{\prime \prime}=y_{1}-t_{2}^{-}
\end{array}
$$

with $t_{1}=x_{1}-y_{1}^{-}-x_{2}+y_{2}^{+}$and $t_{2}=x_{1}+y_{1}^{-}-x_{2}-y_{2}^{+}$.

We can now define an action of $\Sigma_{n}$-words on $\mathbb{Z}^{2 n}$.

Definition 3.10. For $i \in[1, n-1], e= \pm 1$ and $\left(a_{1}, b_{1}, \ldots, a_{n}, b_{n}\right) \in \mathbb{Z}^{2 n}$ we put

$$
\left(a_{1}, b_{1}, \ldots, a_{n}, b_{n}\right) \cdot \sigma_{i}^{e}=\left(a_{1}^{\prime}, b_{1}^{\prime}, \ldots, a_{n}^{\prime}, b_{n}^{\prime}\right)
$$

where $\left(a_{i}^{\prime}, b_{i}^{\prime}, a_{i+1}^{\prime}, b_{i+1}^{\prime}\right)=\left(a_{i}, b_{i}, a_{i+1}, b_{i+1}\right) \cdot \sigma_{i}^{e}$ and $a_{k}^{\prime}=a_{k}, b_{k}^{\prime}=b_{k}$ for $k$ not belonging to $\{i, i+1\}$.

Definition 3.11. For a $\Sigma_{n}$-word $u$ we define $\operatorname{Dyn}(u)$ to be $(0,1, \ldots, 0,1) \cdot u$. Similarly for an $\Sigma_{n}^{*}$-word $v$ we define $\operatorname{Dyn}(v)$ to be $\operatorname{Dyn}(u)$ where $u$ is the $\Sigma_{n}$-word obtained from $u$ using relation (5) of Definition 2.5.

Naturally defined on braid words, Dynnikov's coordinates is a braid invariant.

Proposition 3.12. For all $S_{n}$-words $u$ and $v$ we have $\operatorname{Dyn}(u)=\operatorname{Dyn}(v)$ if and only if $u \equiv v$.

Proof. Direct consequence of Corollary 2.24 page 225 of [15].

We now go back to the problem of testing if a given $S_{n}$-word appears in a set $W$ of $S_{n}$-words. A solution consists in representing the set $W$ in machine by an array. To test if the word $u$ appears in $W$ we can compute $\operatorname{Dyn}(u)$ and compare it to all the values of $\operatorname{Dyn}(v)$ whenever $v$ go through $W$. This method needs at most $1+\operatorname{card}(W)$ computations of Dynnikov's coordinates. If words in $W$ are sorted by their Dynnikov's coordinates we can test if $u$ appear in $W$ using at most $\log _{2}($ card $(W))$ computations of Dynnikov's coordinates. A more efficient solution is obtained using an unordered_set [26] based on a hash function. The insertion and lookup complexity is then constant in average on a RAM machine depending of the hash function. 
As the objective of the current paper is to deepen our knowledge on combinatorics of $B_{4}$, we define a hash function for four strand braids. Assume $\beta$ is a braid of $B_{4}$ given by a $S_{4}$-word $u$. The hash of $\beta$ is

$$
\operatorname{hash}(\beta)=\sum_{i=1}^{4}\left(\operatorname{rem}\left(a_{i}, 256\right) \times 256^{2 i-2}+\operatorname{rem}\left(b_{i}, 256\right) \times 256^{2 i-1}\right),
$$

where $\left(a_{1}, b_{1}, \ldots, a_{4}, b_{4}\right)=\operatorname{Dyn}(u)$ and $\operatorname{rem}(k, 256)$ is the positive remainder of $k$ modulo 256. By construction, $\operatorname{hash}(\beta)$ is an integer lying in $\left[0,2^{64}-1\right]$ and so our hash function is very well suited for 64 bits computers.

3.5. Space complexity. Here again we focus on the case $n=4$. The smallest addressable unit of memory on common computers is the byte which can have 256 different values. As the set $\Sigma_{4}$ has 6 elements we can store three $\Sigma_{4}$-letters using one byte $\left(6^{3}=216\right)$. Hence a $\Sigma_{4}$-word of length $\ell$ requires $\left\lceil\frac{\ell}{3}\right\rceil$ bytes to be stored. Since there are 12 elements in $\Sigma_{4}^{*}$, a $\Sigma_{4}^{*}$-word of length $\ell$ requires $\left\lceil\frac{\ell}{2}\right\rceil$ bytes to be stored.

Assume we want to determine a representative set of $B_{4}\left(\Sigma_{4}, 21\right)$. The memory needed by the algorithm REPSET is at least the space needed to store $\Sigma_{4}$-words of $W_{21}$. By Table 2 of Section 6 there are approximatively $60 \cdot 10^{9}$ elements in this set. With the above storage method of a $\Sigma_{4}$-word, the algorithm needs $7 \cdot 60 \cdot 10^{9}$ bytes, i.e., 391Go of memory to run, which is too much. To reduce the memory requirement we can split the sets $B_{n}\left(S_{n}, \ell\right)$ in many subsets depending of the values of certain braid invariants.

In case we want to determine $g\left(B_{n}, S_{n} ; \ell\right)$ we also store the value of $\omega_{S_{n}}(\bar{u})$ for all words in obtained representative sets.

\section{BRAid template}

Here again $n$ is an integer $\geqslant 2$ and $S_{n}$ denotes either $\Sigma_{n}$ or $\Sigma_{n}^{*}$. Each braid invariant $\iota$ corresponds to a map from $B_{n}$ to a set $X$.

Definition 4.1. A set of braid invariants $\iota_{1}, \ldots, \iota_{m}$ is said to be inductively stable if for every braid $\beta \in B_{n}\left(S_{n}, \ell\right)$ and every $x \in S_{n}$, and every $k=1, \ldots, m$, the value of $\iota_{k}(\beta \cdot x)$ depends only on $\iota_{1}(\beta), \ldots, \iota_{m}(\beta)$ and $x$ but not on $\beta$ itself.

The aim of this section is to determine an inductively stable set of braid invariants in order to split in many pieces the determination of a representative set of $B_{n}\left(S_{n}, \ell\right)$.

4.1. Permutation. For $n \geqslant 2$ we denote by $\mathfrak{S}_{n}$ the set of all bijections of $\{1, \ldots, n\}$ into itself. The transposition $(i i+1)$ of $\mathfrak{S}_{n}$ exchanging $i$ and $i+1$ is denoted $s_{i}$.

Definition 4.2. We denote by $\pi: B_{n} \rightarrow \mathfrak{S}_{n}$ the surjective homomorphism of $\left(B_{n}, \cdot\right)$ to $\left(\mathfrak{S}_{n}, \circ\right)$ defined by $\pi\left(\sigma_{i}\right)=s_{i}$.

If $\beta$ is a braid of $B_{n}$ then $\pi(\beta)$ is the permutation of $\mathfrak{S}_{n}$ such that the strand ending at position $i$ starts at position $\pi(\beta)(i)$.

Example 4.3. For $\beta=\sigma_{1} \sigma_{2}^{-1} \sigma_{1} \sigma_{2}$ we have $\pi(\beta)=s_{1} s_{2} s_{1} s_{2}=\left(\begin{array}{ccc}1 & 2 & 3 \\ 3 & 1 & 2\end{array}\right)$, as illustrated on the following diagram : 


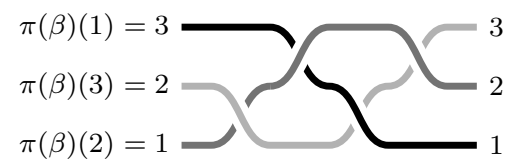

As $\pi$ is a homomorphism, for all $\beta \in B_{n}$ and $x \in S_{n}$ we have $\pi(\beta \cdot x)=\pi(\beta) \circ \pi(x)$ and so the singleton $\{\pi\}$ is inductively stable.

Lemma 4.4. For $1 \leqslant p<q \leqslant n$ we have $\pi\left(a_{p, q}\right)=(p q)$.

Proof. As $\pi$ is a homomorphism, Definition 2.5 gives

$$
\begin{aligned}
\pi\left(a_{p, q}\right) & =\pi\left(\sigma_{p}\right) \circ \ldots \circ \pi\left(\sigma_{q-1}\right) \circ \pi\left(\sigma_{q-2}\right)^{-1} \circ \ldots \circ \pi\left(\sigma_{p}\right)^{-1} \\
& =(p p+1) \circ \ldots \circ(q-1 q) \circ(q-2 q-1) \circ \ldots \circ(p p+1) \\
& =(p q) .
\end{aligned}
$$

4.2. Linking numbers. Assume $\beta$ is a braid of $B_{n}$ and let $i$ and $j$ be two different integers of $[1, n]$. The linking number of the two strands $i$ and $j$ in $\beta$ is the algebraic number of crossings in $\beta$ involving the strands $i$ and $j$. A positive crossing $\left(\sigma_{k}\right)$ counts for +1 whereas a negative one $\left(\sigma_{k}^{-1}\right)$ counts for -1 :

$$
\left.\mathcal{Y}_{\rightarrow+1}\right)
$$

Definition 4.5. For $\beta \in B_{n}$ and $i, j$ two different integers of $[1, n]$ we denote by $\ell_{i, j}(\beta)$ the linking number of strands $i$ and $j$ in $\beta$. The map $\ell_{i, j}: B_{n} \rightarrow \mathbb{Z}$ is then a braid invariant.

A priori, our definition of linking numbers depends of a diagram coding the braid and not on the braid itself. An immediate argument using relations (1) and (2) guarantees this is not the case. The reader can consult [12] page 29 for a more formal definition of linking number ${ }^{1}$ based of an integral definition and a geometric realization of $\beta$ in $\mathbb{R}^{3}$.

Lemma 4.6. Let $i, j$ be two integers satisfying $1 \leqslant i<j \leqslant n$ and $e= \pm 1$.

- For all $k \in[1, n-1]$ we have

$$
\ell_{i, j}\left(\sigma_{k}^{e}\right)= \begin{cases}e & \text { if } i=k \text { and } j=k+1, \\ 0 & \text { otherwise. }\end{cases}
$$

- For all $1 \leqslant p<q \leqslant n$ we have

$$
\ell_{i, j}\left(a_{p, q}^{e}\right)= \begin{cases}e & \text { if } i=p \text { and } j=q, \\ 1 & \text { if } i=p \text { and } j<q, \\ -1 & \text { if } p<i \text { and } j=q, \\ 0 & \text { otherwise. }\end{cases}
$$

Proof. The case of $\sigma_{k}^{e}$ is immediate. The different values of $\ell_{i, j}\left(a_{p, q}^{e}\right)$ can be obtained from the following diagram of $a_{p, q}^{e}=\sigma_{p} \ldots \sigma_{q-2} \sigma_{q-1}^{e} \sigma_{q-2}^{-1} \ldots \sigma_{p}^{-1}$ :

\footnotetext{
${ }^{1}$ In fact, the two definitions are slightly different but we have $\ell_{i, j}(\beta)=2 \lambda_{i, j}(\beta)$.
} 


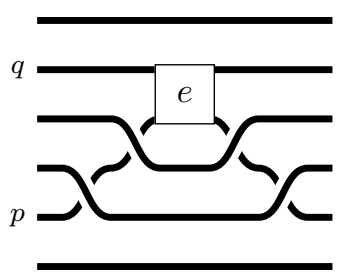

Lemma 4.7. For $\beta$ and $\gamma$ two braids of $B_{n}$ and $1 \leqslant i<j \leqslant n$ we have

$$
\ell_{i, j}(\beta \cdot \gamma)=\ell_{i, j}(\beta)+\ell_{\pi(\beta)^{-1}(i), \pi(\beta)^{-1}(j)}(\gamma),
$$

with the convention $\ell_{p, q}=\ell_{q, p}$ for $p>q$.

Proof. Immediate as soon as we consider the following diagram :

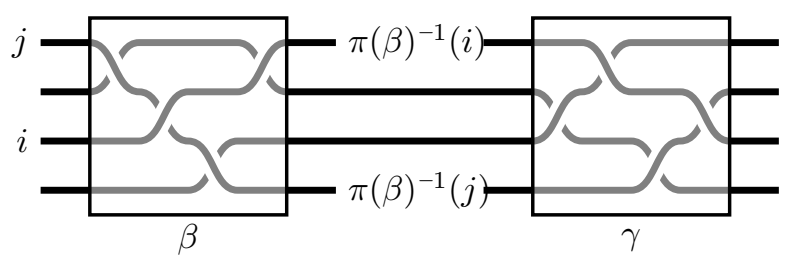

Corollary 4.8. The set of invariants $\{\pi\} \cup\left\{\ell_{i, j}, 1 \leqslant i<j \leqslant n\right\}$ is inductively stable.

Proof. A direct consequence of Lemma 4.7 together with the fact that $\pi$ is a homomorphism.

4.3. Template. We now introduce the notion of template of a braid which will be used to parallelize the determination of a representative set of $B_{n}\left(S_{n}, \ell\right)$.

Definition 4.9. The template of a braid $\beta \in B_{n}$ is the tuple

$$
\tau(\beta)=\left(\pi(\beta), \ell_{1,2}(\beta), \ell_{1,3}(\beta), \ell_{2,3}(\beta), \ldots, \ell_{1, n}(\beta), \ldots, \ell_{n-1, n}(\beta)\right) \in \mathfrak{S}_{n} \times \mathbb{Z}^{\frac{n(n-1)}{2}},
$$

where integer $\ell_{i, j}(\beta)$ appears before $\ell_{r, s}(\beta)$ whenever $(i, j)$ is smaller than $(r, s)$ with respect to the the co-lexicographic ordering on $\mathbb{N}^{2}:(i, j)<(p, q)$ if $j<q$ or if $j=q$ and $i<p$. For a braid template $t$ we denote by $t[\pi]$, resp. $t\left[\ell_{i, j}\right]$ the corresponding component. For $\ell \in \mathbb{N}$ we denote by $T_{n}\left(S_{n}, \ell\right)$ the set $\left\{\tau(\beta), \beta \in B_{n}\left(S_{n}, \ell\right)\right\}$ and by $T_{n}$ the set $\left\{\tau(\beta), \beta \in B_{n}\right\}$ of all templates on $B_{n}$.

Lemma 4.10. For all $\beta \in B_{n}$ and all $x \in S_{n}$, the template $\tau(\beta) * x=\tau(\beta \cdot x)$ depends only on $\tau(\beta)$ and $x$.

Proof. A direct consequence of Corollary 4.8 and Definition 4.9.

Example 4.11. Let $t$ be a template of $T_{3}$ with $t[\pi]$ the cycle $\left(\begin{array}{lll}1 & 3 & 2\end{array}\right)$. Let us compute the template $t * a_{1,3}^{-1}$. We write $t=\left(\pi, \ell_{1,2}, \ell_{1,3}, \ell_{2,3}\right)$. The inverse of $\pi$ is the cycle $\left(\begin{array}{lll}1 & 2 & 3\end{array}\right)$ and so we obtain $\pi^{-1}(\{1,2\})=\{2,3\}, \pi^{-1}(\{1,3\})=\{1,2\}$ and $\pi^{-1}(\{2,3\})=\{1,3\}$. Eventually, from $\ell_{1,2}\left(a_{1,3}^{-1}\right)=1, \ell_{1,3}\left(a_{1,3}^{-1}\right)=-1$ and $\ell_{2,3}\left(a_{1,3}^{-1}\right)=-1$ we obtain

$$
\begin{aligned}
t * a_{1,3}^{-1} & =\left(\left(\begin{array}{lll}
1 & 3 & 2
\end{array}\right) \circ\left(\begin{array}{ll}
1 & 3
\end{array}\right), \ell_{1,2}+\ell_{2,3}\left(a_{1,3}^{-1}\right), \ell_{1,3}+\ell_{1,2}\left(a_{1,3}^{-1}\right), \ell_{2,3}+\ell_{1,3}\left(a_{1,3}^{-1}\right)\right) \\
& =\left(\left(\begin{array}{ll}
1 & 2
\end{array}\right), \ell_{1,2}-1, \ell_{1,3}+1, \ell_{2,3}-1\right) .
\end{aligned}
$$


Definition 4.12. For $\ell \in \mathbb{N}$ and $t \in T_{n}$ we denote by $B_{n}\left(S_{n}, \ell, t\right)$ the set of all braids of $B_{n}$ with $S_{n}$-length $\ell$ and template $t$.

By very definitions we have

$$
B_{n}\left(S_{n}, \ell\right)=\bigsqcup_{t \in T_{n}\left(S_{n}, \ell\right)} B_{n}\left(S_{n}, \ell, t\right) .
$$

Algorithm 2 - TEMPRepSet is a "template" version of Algorithm 1 - RePSET for which we assume we dispose of a function $\operatorname{LOAD}(n, \ell, t)$ loading a representative set of $B_{n}\left(S_{n}, \ell, t\right)$ from a storage memory like a hard disk. We also assume we have a function $\operatorname{SAVE}(W, n, \ell, t)$ saving a representative set of $B_{n}\left(S_{n}, \ell, t\right)$ to that storage memory.

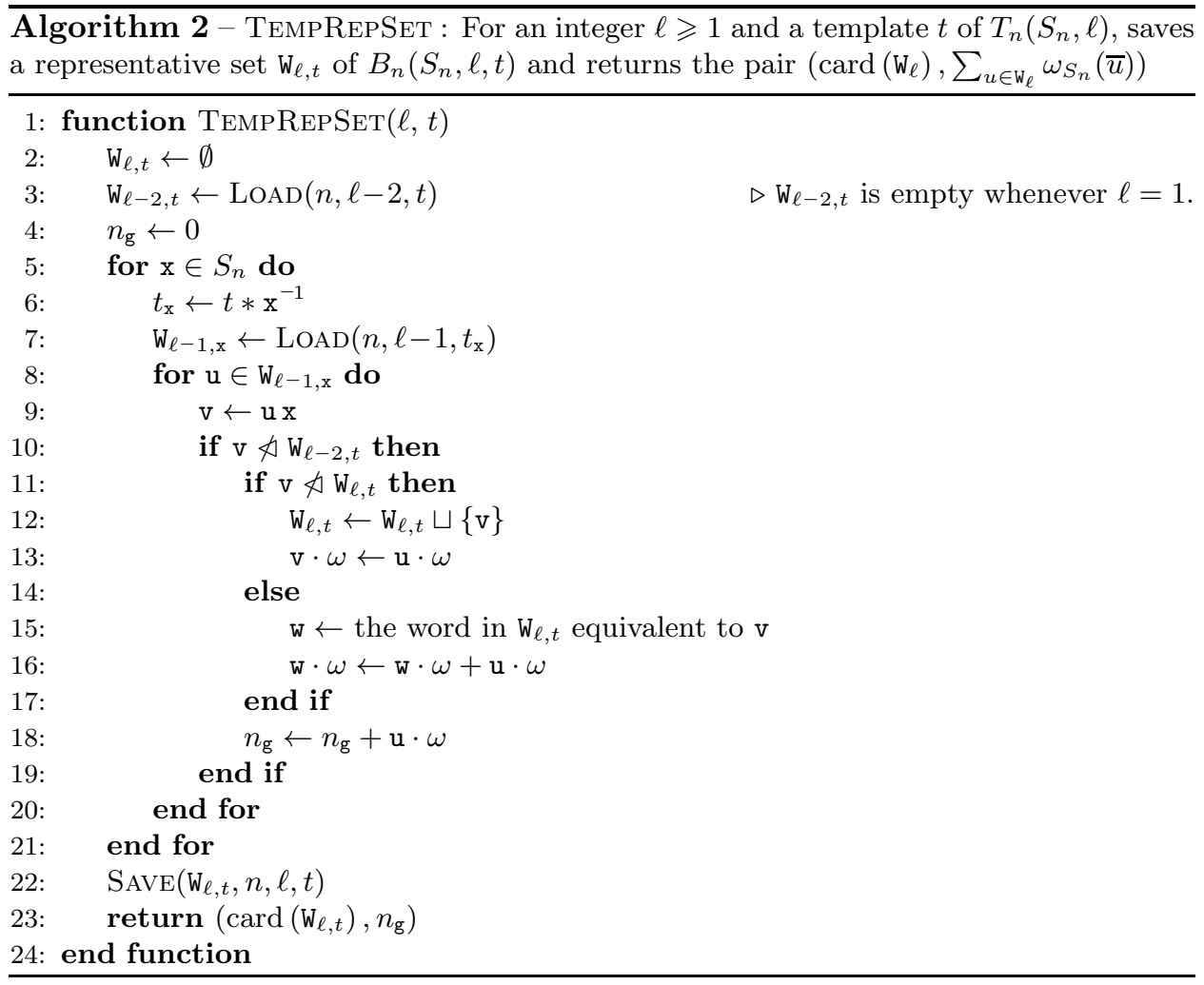

In order to compute a representative set of $B_{n}\left(S_{n}, \ell\right)$ using Algorithm TEMPREPSET we must first compute the template set $T_{n}\left(S_{n}, \ell\right)$. From inclusion (9) we obtain

$$
T_{n}\left(S_{n}, \ell\right) \subseteq\left\{t * x \text { for }(t, x) \in T_{n}\left(S_{n}, \ell-1\right) \times S_{n}\right\} .
$$

A template $t$ from the set in the right-hand side of (13) belongs to $T_{n}\left(S_{n}, \ell\right)$ if and only if there exists a braid $\beta \in B_{n}\left(S_{n}, \ell\right)$ such that $\tau(\beta)=t$. Hence a full run consists in calling the function TEMPREPSET for each template $t$ from the set in the right-hand side of (13). Such a template $t$ will belongs to $T_{n}\left(S_{n}, \ell\right)$ if and only if the returned value is different from $(0,0)$. Putting all pieces together we obtain : 


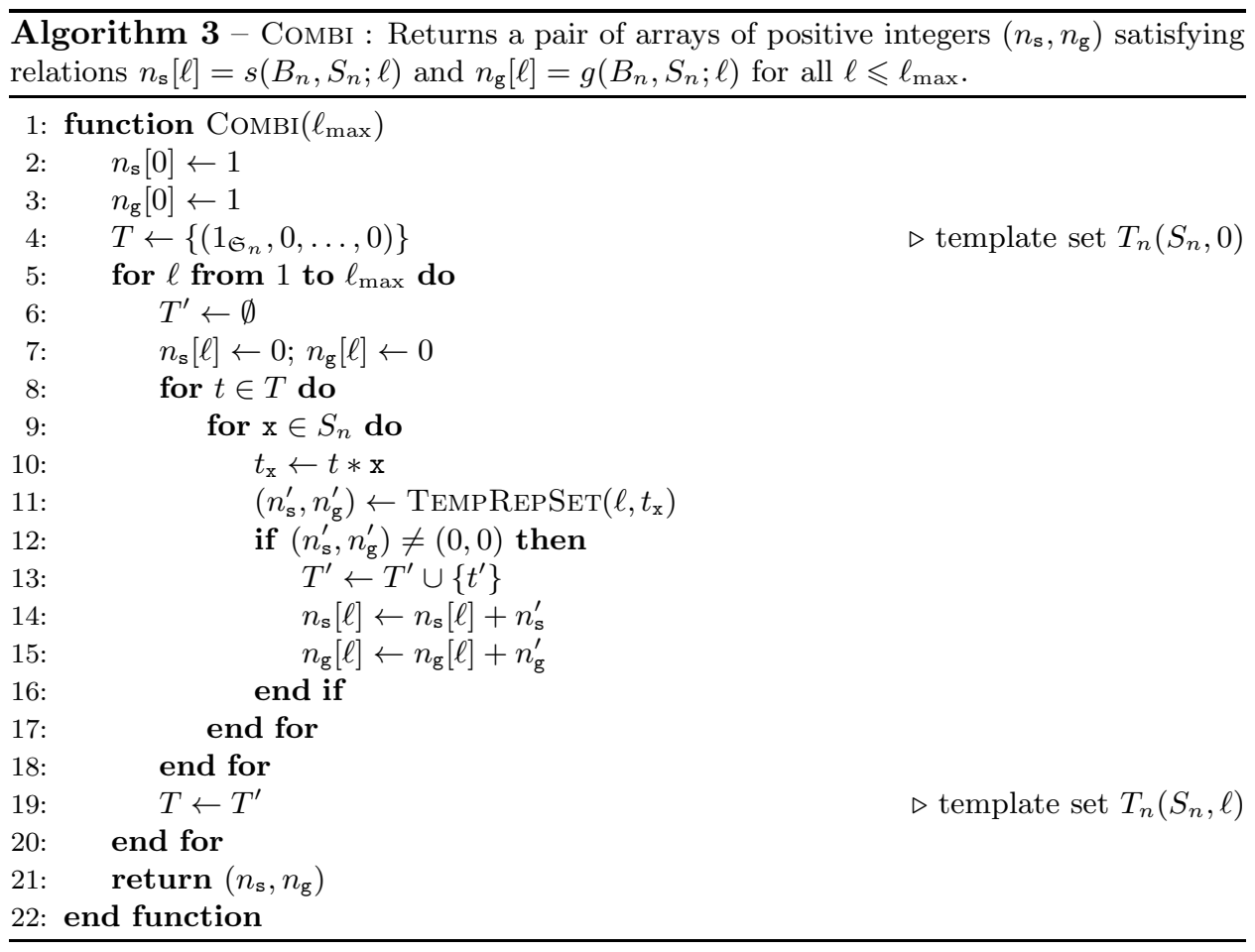

\section{REDUCED BRAid TEMPlates}

Here again $n$ is an integer $\geqslant 2$ and $S_{n}$ denotes either $\Sigma_{n}$ or $\Sigma_{n}^{*}$. Experiments using Algorithm 2 - TEMPREPSET suggest that some sets $B_{n}\left(S_{n}, \ell, t\right)$ are in bijection for a given $\ell$. We can use this fact to improve the efficiency of Algorithm 3 - ComBI and reduce the needed storage space.

\subsection{Stable word maps.}

Definition 5.1. A bijection $\mu$ of the set of $S_{n}$-words is $S_{n}$-stable if

$-i$ ) for all $S_{n}$-word $w$ we have $|\mu(w)|=|w|$;

- ii) for all $S_{n}$-words $u$ and $v$ we have $\mu(u) \equiv \mu(v) \Leftrightarrow u \equiv v$;

- iii) for all $S_{n}$-word $u$ the template $\tau(\overline{\mu(u)})$ depends only on $\tau(\bar{u})$.

For such a $S_{n}$-stable map $\mu$ we denote by $\mu^{T}$ the map of $T_{n}$ defined by

$$
\mu^{T}(t)=\tau(\overline{\mu(u)})
$$

where $u$ is any $S_{n}$-word satisfying $\tau(\bar{u})=t$. We also define a bijection $\bar{\mu}$ of $B_{n}$ by

$$
\bar{\mu}(\beta)=\overline{\mu(u)}
$$

where $u$ is any $S_{n}$-word satisfying $\bar{u}=\beta$.

Whenever $\mu$ is $S_{n}$-stable, Condition $\mathrm{iii}$ ) of Definition 5.1 guarantees that the template of the image by $\bar{\mu}$ of a braid $\beta$ does not depend on $\beta$ but on its template $t$ and so $\mu^{T}$ is well defined.

Lemma 5.2. For every $S_{n}$-stable bijection $\mu$, we have

- i) $\mu^{T}$ is a permutation of $T_{n}$,

- ii) a $S_{n}$-word $u$ is geodesic if and only if $\mu(u)$ is. 
Proof. By $i$ ) and $i i)$ of Definition 5.1 we obtain that $\mu$ induces a permutation on the finite set $B_{n}\left(S_{n}, \ell\right)$. It follows that $\mu^{T}$ induces a permutation on $T_{n}\left(S_{n}, \ell\right)$. For a template $t$ of $T_{n}$ there exists an integer $\ell \in \mathbb{N}$ such that $t$ belongs to $T_{n}\left(S_{n}, \ell\right)=$ $\mu^{T}\left(T_{n}\left(S_{n}, \ell\right)\right)$ and so $\mu^{T}$ is surjective. We now prove the injectivity. For $t \in T_{n}$, we denote by $\lambda(t)$ the minimal integer $\ell$ such that $t$ belongs to $T_{n}\left(S_{n}, \ell\right)$. Since $\mu^{T}$ induces a permutation on $T_{n}\left(S_{n}, \ell\right)$ for all $\ell$ we have $\lambda\left(\mu^{T}(t)\right)=\lambda(t)$. Let $t$ and $t^{\prime}$ be two templates of $T_{n}$ satisfying $\mu^{T}(t)=\mu^{T}\left(t^{\prime}\right)$. By the above we have

$$
\lambda(t)=\lambda\left(\mu^{T}(t)\right)=\lambda\left(\mu^{T}\left(t^{\prime}\right)\right)=\lambda\left(t^{\prime}\right),
$$

and so there exists $\ell$ such that $t$ and $t^{\prime}$ belong to $T_{n}\left(S_{n}, \ell\right)$. Since $\mu^{T}$ induces a permutation on $T_{n}\left(S_{n}, \ell\right)$ we obtain $t=t^{\prime}$, proving the injectivity of $\mu^{T}$.

Let us now prove $i i)$. Let $u$ be a $S_{n}$-word. If the word $v=\mu(u)$ is not geodesic then there exists a strictly shorter $S_{n}$-word $v^{\prime}$ equivalent to $v$. As $\mu$ is a bijection we put $u^{\prime}=\mu^{-1}\left(v^{\prime}\right)$. We obtain $\mu(u)=v \equiv v^{\prime}=\mu\left(u^{\prime}\right)$. From conditions $\left.i i\right)$ and $i$ ) of Definition 5.1 we have $u \equiv u^{\prime}$ together with $|u|=|v|>\left|v^{\prime}\right|=\left|u^{\prime}\right|$ and so $u$ is not geodesic. A similar argument establishes the converse implication.

5.2. Examples. Let us now introduce some useful examples of $S_{n}$-stable bijections. Eventually such a $S_{n}$-stable bijection $\mu$ will be used to obtain a representative set of $T_{n}\left(S_{n}, \ell, \mu^{T}(t)\right)$ from a representative set of $T_{n}\left(S_{n}, \ell, t\right)$. This is why it is necessary to specify how to obtain $\mu^{T}(t)$ from $t$ in propositions 5.3, 5.4, 5.8 and 5.13. However the reader may choose to ignore these parts without affecting the understanding of the rest ot the article.

\subsubsection{First examples.}

Proposition 5.3. The map $\operatorname{inv}_{S_{n}}$ of $S_{n}$-words defined by

$$
\operatorname{inv}_{S_{n}}\left(x_{1} \cdots x_{t}\right)=x_{t}^{-1} \cdots x_{1}^{-1}
$$

is $S_{n}$-stable. Moreover for every template $t \in T_{n}$ we have

$$
\operatorname{inv}_{S_{n}}^{T}(t)[\pi]=t[\pi]^{-1} \quad \text { and } \operatorname{inv}_{S_{n}}^{T}(t)\left[\ell_{i, j}\right]=-t\left[\ell_{t[\pi](i), t[\pi](j)}\right] \text { for } 1 \leqslant i<j \leqslant n .
$$

Proof. Condition $i$ ) of Definition 5.1 is immediate. For two $S_{n}$-words $u$ and $v$, the relation $u \equiv v$ is equivalent to $v^{-1} u \equiv \varepsilon$ which is itself equivalent to $v^{-1} \equiv u^{-1}$, hence Condition ii) is established. Let $u$ be a $S_{n}$-word and $v$ be $\operatorname{inv}_{S_{n}}(u)$. By definition, we have $\bar{v}=\bar{u}^{-1}$. Since $\pi$ is a homomorphism we have $\pi(\bar{v})=\pi(\bar{u})^{-1}$. Let $1 \leqslant i<j \leqslant n$ be two integers. From $1=\overline{v u}$, Lemma 4.7 implies

$$
0=\ell_{i, j}(1)=\ell_{i, j}(\bar{v})+\ell_{\pi(\bar{u})(i), \pi(\bar{u})(j)}(\bar{u})
$$

and so $\ell_{i, j}(\bar{v})=-\ell_{\pi(\bar{u})(i), \pi(\bar{u})(j)}(\bar{u})$. Therefore Condition $\left.i i i\right)$ is also satisfied.

We now point out a divergence between the Artin and dual presentations of the braid group $B_{n}$.

Proposition 5.4. For $n \geqslant 3$, the map of $S_{n}$-words $\theta_{S_{n}}$ defined by

$$
\theta_{S_{n}}\left(x_{1} \cdots x_{t}\right)=x_{1}^{-1} \cdots x_{t}^{-1}
$$

is $S_{n}$-stable if and only if $S_{n}=\Sigma_{n}$. Moreover for every template $t \in T_{n}$ we have

$$
\theta_{\Sigma_{n}}^{T}(t)[\pi]=t[\pi] \quad \text { and } \quad \theta_{\Sigma_{n}}^{T}(t)\left[\ell_{i, j}\right]=-t\left[\ell_{i, j}\right] \text { for } 1 \leqslant i<j \leqslant n .
$$


Proof. By construction, Condition $i$ ) of Definition 5.1 is satisfied. Let us verify Condition $i$ ) for $\theta_{\Sigma_{n}}$. It is sufficient to prove $\theta(u) \equiv \theta(v)$ whenever $u=v$ is a relation of the Artin's semigroup presentation of $B_{n}$. Let $i \in[1, n-1]$. We have $\theta\left(\sigma_{i} \sigma_{i}^{-1}\right)=\sigma_{i}^{-1} \sigma_{i} \equiv \varepsilon, \theta\left(\sigma_{i}^{-1} \sigma_{i}\right)=\sigma_{i} \sigma_{i}^{-1} \equiv \varepsilon$ and so we get

$$
\theta\left(\sigma_{i} \sigma_{i}^{-1}\right)=\theta\left(\sigma_{i}^{-1} \sigma_{i}\right)=\theta(\varepsilon) .
$$

Assume now $i$ and $j$ are integers of $[1, n-1]$ satisfying $|i-j| \geqslant 2$. From $\sigma_{i} \sigma_{j} \equiv \sigma_{j} \sigma_{i}$ we obtain successively

$$
\sigma_{j}^{-1} \sigma_{i} \sigma_{j} \equiv \sigma_{i}, \quad \sigma_{j}^{-1} \sigma_{i} \equiv \sigma_{i} \sigma_{j}^{-1}, \quad \sigma_{i}^{-1} \sigma_{j}^{-1} \sigma_{i} \equiv \sigma_{j}^{-1}, \quad \sigma_{i}^{-1} \sigma_{j}^{-1} \equiv \sigma_{j}^{-1} \sigma_{i}^{-1},
$$

and so $\theta\left(\sigma_{i} \sigma_{j}\right)=\sigma_{i}^{-1} \sigma_{j}^{-1} \equiv \sigma_{j}^{-1} \sigma_{i}^{-1}=\theta\left(\sigma_{j} \sigma_{i}\right)$. A similar sequence of equivalences implies $\theta\left(\sigma_{i} \sigma_{j} \sigma_{i}\right) \equiv \theta\left(\sigma_{j} \sigma_{i} \sigma_{j}\right)$ for $i, j$ in $[1, n-1]$ with $|i-j| \leqslant 1$.

Let $u$ be an $\Sigma_{n}$-word. For $x \in \Sigma_{n}$, the permutation $\pi(\bar{x})$ is a transposition and so the relation $\pi(\bar{x})=\pi\left(\overline{x^{-1}}\right)$ holds. Hence we obtain $\pi\left(\overline{\theta_{\Sigma_{n}}(u)}\right)=\pi(\bar{u})$. We denote by $u_{k}$ the prefix of $u$ of length $k$. An immediate induction on $k$, together with $\pi\left(\overline{\theta_{\Sigma_{n}}\left(u_{k}\right)}\right)=\pi\left(\overline{u_{k}}\right)$ and Lemma 4.7 establish $\ell_{i, j}\left(\overline{\theta_{\Sigma_{n}}(u)}\right)=-\ell_{i, j}(\bar{u})$. Condition $i$ ii) is then satisfied by $\theta_{\Sigma_{n}}$.

Let us focus now on the map $\theta_{\Sigma_{n}^{*}}$. In $B_{4}$ we have the relation $a_{1,2} a_{2,3} \equiv a_{2,3} a_{1,3}$ while $a_{1,2}^{-1} a_{2,3}^{-1}$ is not equivalent to $a_{2,3}^{-1} a_{1,3}^{-1}$ as shown by the following diagrams.

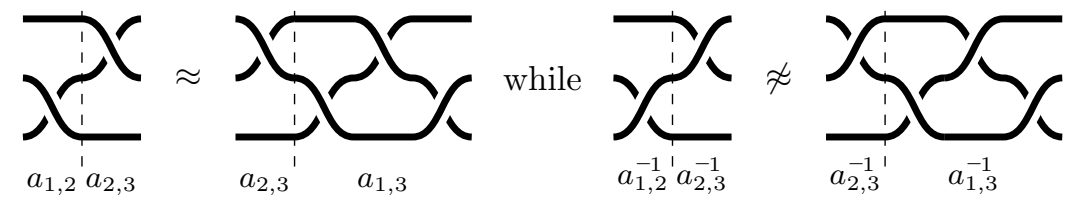

The non isotopy of the two right-most diagrams can be established evaluating $\ell_{1,3}$ for example. Indeed we have $\ell_{1,3}\left(a_{1,2}^{-1} a_{2,3}^{-1}\right)=-1$ and $\ell_{1,3}\left(a_{2,3}^{-1} a_{1,3}^{-1}\right)=1$.

5.2.2. Garside homorphisms. We now consider the "word version" of the classical and dual Garside automorphisms of $B_{n}$.

Definition 5.5. The Garside automorphism of $B_{n}$ is $\bar{\Phi}_{n}(\beta)=\Delta_{n} \beta \Delta_{n}^{-1}$ where $\Delta_{n}$ is given by $\Delta_{2}=\sigma_{1}$ and $\Delta_{k}=\sigma_{1} \cdots \sigma_{k-1} \Delta_{k-1}$ for $k \geqslant 3$.

For example we have $\Delta_{4}=\sigma_{1} \sigma_{2} \sigma_{3} \cdot \Delta_{3}=\sigma_{1} \sigma_{2} \sigma_{3} \cdot \sigma_{1} \sigma_{2} \cdot \Delta_{2}=\sigma_{1} \sigma_{2} \sigma_{3} \cdot \sigma_{1} \sigma_{2} \cdot \sigma_{1}$, which corresponds to the following diagram:

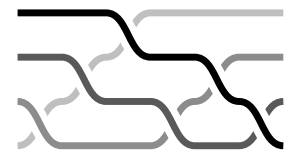

For all $k \in[1, n]$ we have:

$$
\pi\left(\Delta_{n}\right)(k)=n+1-k .
$$

As we can notice in the previous diagram, the braid $\Delta_{n}$ can be represented by a diagram in which each two strands cross exactly once implying

$$
\ell_{i, j}\left(\Delta_{n}\right)=1 \text { and } \ell_{i, j}\left(\Delta_{n}^{-1}\right)=-1 \quad \text { for all } 1 \leqslant i<j \leqslant n .
$$

The result involving $\Delta_{n}^{-1}$ is a direct consequence of that of $\Delta_{n}$ together with Proposition 5.3. The following lemma is a well-known result about the Garside automorphism $\bar{\Phi}_{n}$. 
Lemma 5.6. For $n \geqslant 3$, the automorphism $\bar{\Phi}_{n}$ has order 2 and for every integer $k$ in $[1, n-1]$ we have $\bar{\Phi}_{n}\left(\sigma_{k}\right)=\sigma_{n-k}$.

Proof. Let $k \in[1, n-1]$. Relation $\bar{\Phi}_{n}\left(\sigma_{k}\right)=\sigma_{n-k}$ is an easy verification from the Artin presentation of $B_{n}$ (see Lemma I.3.6 of [12]). We conclude with $\bar{\Phi}_{n}^{2}\left(\sigma_{k}\right)=$ $\bar{\Phi}_{n}\left(\sigma_{n-k}\right)=\sigma_{n-(n-k)}=\sigma_{k}$.

Definition 5.7. We denote by $\Phi_{n}$ the homomorphism of $\Sigma_{n}$-words defined for every integer $k$ in $[1, n]$ by $\Phi_{n}\left(\sigma_{k}\right)=\sigma_{n-k}$.

By Lemma 5.6 , for every $\Sigma_{n}$-word $u$ we have

$$
\overline{\Phi_{n}(u)}=\bar{\Phi}_{n}(\bar{u})=\Delta_{n} \bar{u} \Delta_{n}^{-1} .
$$

Proposition 5.8. The map $\Phi_{n}$ is $\Sigma_{n}$-stable. Moreover for every template $t \in T_{n}$ we have:

$$
\begin{array}{r}
\left(\Phi_{n}^{T}(t)[\pi]\right)(k)=n+1-t[\pi](n+1-k) \quad \text { for all } k \in[1, n] \\
\Phi_{n}^{T}(t)\left[\ell_{i, j}\right]=t\left[\ell_{n+1-j, n+1-i}\right] \quad \text { for all } 1 \leqslant i<j \leqslant n .
\end{array}
$$

Proof. Condition $i$ ) and $i$ ) of Definition 5.1 are easily established since $\Phi_{n}$ is defined from the conjugation $\bar{\Phi}_{n}$ which induces a bijection on $\Sigma_{n}$. We now prove Condition iii) of Definition 5.1. Let $u$ be a $\Sigma_{n}$-word. By (16) we have

$$
\pi\left(\overline{\Phi_{n}(u)}\right)=\pi\left(\Delta_{n}\right) \circ \pi(\bar{u}) \circ \pi\left(\Delta_{n}\right)^{-1}=\pi\left(\Delta_{n}\right) \circ \pi(\bar{u}) \circ \pi\left(\Delta_{n}\right) .
$$

Relation (14) implies that for every integer $k \in[1, n]$ we have

$$
\pi\left(\overline{\Phi_{n}(u)}\right)(k)=n+1-\pi(\bar{u})(n+1-k) .
$$

Let $1 \leqslant i<j \leqslant n$ be two integers. Lemma 4.7 together with (15) give

$$
\begin{aligned}
\ell_{i, j}\left(\overline{\Phi_{n}(u)}\right)=\ell_{i, j}\left(\Delta_{n} \bar{u} \Delta_{n}^{-1}\right) & =\ell_{i, j}\left(\Delta_{n} \bar{u}\right)+\ell_{\pi\left(\Delta_{n} \bar{u}\right)^{-1}(i), \pi\left(\Delta_{n} \bar{u}\right)^{-1}(j)}\left(\Delta_{n}^{-1}\right) \\
& =\ell_{i, j}\left(\Delta_{n} \bar{u}\right)-1 \\
& =\ell_{i, j}\left(\Delta_{n}\right)+\ell_{\pi\left(\Delta_{n}\right)^{-1}(i), \pi\left(\Delta_{n}\right)^{-1}(j)}(\bar{u})-1 \\
& =\ell_{\pi\left(\Delta_{n}\right)^{-1}(i), \pi\left(\Delta_{n}\right)^{-1}(j)}(\bar{u}) \\
& =\ell_{n+1-i, n+1-j}(\bar{u})=\ell_{n+1-j, n+1-i}(\bar{u}) .
\end{aligned}
$$

Definition 5.9. The dual Garside automorphism of $B_{n}$ is $\bar{\phi}_{n}(\beta)=\delta_{n} \beta \delta_{n}^{-1}$ where $\delta_{n}$ is given by $\delta_{n}=a_{1,2} \cdots a_{n-1, n}=\sigma_{1} \cdots \sigma_{n-1}$.

For example we have $\delta_{4}=\sigma_{1} \sigma_{2} \sigma_{3}$ and $\delta_{4}^{-1}=\sigma_{3}^{-1} \sigma_{2}^{-1} \sigma_{1}^{-1}$ which correspond to the following diagrams:

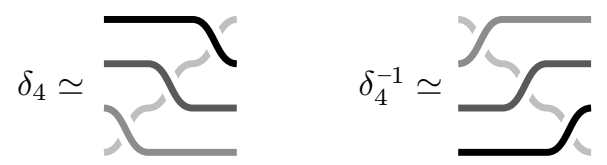

Notation 5.10. For all $n \in \mathbb{N}$ and $k \in[0, n+1]$ we put

$$
[k]_{n}= \begin{cases}1 & \text { if } k=n+1 \\ n & \text { if } k=0 \\ k & \text { otherwise }\end{cases}
$$

Moreover for all integers $i$ and $j$ the symbol $\mathbf{1}_{i=j}$ equals 1 if the relation $i=j$ holds and 0 otherwise. 
As we can directly see on diagrams of (18), for all $k \in[1, n]$ we have

$$
\pi\left(\delta_{n}\right)(k)=[k+1]_{n} \text { and } \pi\left(\delta_{n}^{-1}\right)(k)=[k-1]_{n},
$$

moreover for all $1 \leqslant i<j \leqslant n$ we have

$$
\ell_{i, j}\left(\delta_{n}\right)=\mathbf{1}_{i=1} \quad \text { and } \quad \ell_{i, j}\left(\delta_{n}^{-1}\right)=-\mathbf{1}_{j=n} .
$$

Lemma 5.11. The automorphism $\bar{\phi}_{n}$ has order $n$ and for all $1 \leqslant p<q \leqslant n$ we have

$$
\bar{\phi}_{n}\left(a_{p, q}\right)=a_{[p+1]_{n},[q+1]_{n}}
$$

with the convention $a_{i, j}=a_{j, i}$ whenever $j>i$ holds.

Proof. Computation of $\bar{\phi}_{n}\left(a_{p, q}\right)$ is an easy verification from Birman-Ko-Lee's presentation of $B_{n}$. The result on the order of $\bar{\phi}_{n}$ is then an immediate consequence.

Definition 5.12. We denote by $\phi_{n}$ the homomorphism of $\Sigma_{n}^{*}$-words defined for all integers $p$ and $q$ with $1 \leqslant p<q \leqslant n$ by

$$
\phi_{n}\left(a_{p, q}\right)=a_{[p+1]_{n},[q+1]_{n}}
$$

By Lemma 5.11 , for every $\Sigma_{n}^{*}$-word $u$ we have

$$
\overline{\phi_{n}(u)}=\bar{\phi}_{n}(\bar{u})=\delta_{n} \bar{u}^{-1} \delta_{n}^{-1} \text {. }
$$

Proposition 5.13. The map $\phi_{n}$ is $\Sigma_{n}^{*}$-stable. Moreover for every template $t \in T_{n}$ we have

$$
\begin{aligned}
& \left(\phi_{n}^{T}(t)[\pi]\right)(k)=\left[1+t[\pi]\left([k-1]_{n}\right)\right]_{n} \quad \text { for all } k \in[1, n], \\
& \phi_{n}^{T}(t)\left[\ell_{i, j}\right]=t\left[\ell_{[i-1]_{n},[j-1]_{n}}\right]+\mathbf{1}_{i=1}-\mathbf{1}_{[1+t[\pi](n)]_{n}=j} \quad \text { for all } 1 \leqslant i<j \leqslant n .
\end{aligned}
$$

Proof. The proof is similar to that of Proposition 5.8. We detail only the case of Condition $i i i$ ). Let $u$ be a $\Sigma_{n}^{*}$-word and $k$ be in $[1, n]$. From (21) and (19) we obtain

$$
\begin{aligned}
\pi\left(\overline{\phi_{n}(u)}\right)(k) & =\pi\left(\delta_{n}\right)\left(\pi(\bar{u})\left(\pi\left(\delta_{n}\right)^{-1}(k)\right)\right) \\
& =\pi\left(\delta_{n}\right)\left(\pi(\bar{u})\left([k-1]_{n}\right)\right) \\
& =\left[1+\pi(\bar{u})\left([k-1]_{n}\right)\right]_{n} .
\end{aligned}
$$

Let $1 \leqslant i<j \leqslant n$ be two integers. Lemma 4.7 implies

$$
\ell_{i, j}\left(\overline{\phi_{n}(u)}\right)=\ell_{i, j}\left(\delta_{n} \cdot \bar{u} \cdot \delta_{n}^{-1}\right)=\ell_{i, j}\left(\delta_{n} \cdot \bar{u}\right)+\ell_{\pi\left(\delta_{n} \cdot \bar{u}\right)^{-1}(i), \pi\left(\delta_{n} \cdot \bar{u}\right)^{-1}(j)}\left(\delta_{n}^{-1}\right) .
$$

From (20) we get that $\ell_{\pi\left(\delta_{n} \cdot \bar{u}\right)^{-1}(i), \pi\left(\delta_{n} \cdot \bar{u}\right)^{-1}(j)}\left(\delta_{n}^{-1}\right)$ is non zero iff $\pi\left(\delta_{n} \cdot \bar{u}\right)^{-1}(j)=n$, i.e., iff $\pi\left(\delta_{n} \cdot \bar{u}\right)(n)=j$ which is equivalent to $[1+\pi(\bar{u})(n)]_{n}=j$. We then obtain

$$
\begin{aligned}
\ell_{i, j}\left(\overline{\phi_{n}(u)}\right) & =\ell_{i, j}\left(\delta_{n} \cdot \bar{u}\right)+ \begin{cases}-1 & \text { if }[1+\pi(\bar{u})(n)]_{n}=j, \\
0 & \text { otherwise, }\end{cases} \\
& =\ell_{i, j}\left(\delta_{n} \cdot \bar{u}\right)-\mathbf{1}_{[1+\pi(\bar{u})(n)]_{n}=j .}
\end{aligned}
$$

Moreover, by (20) we have

$$
\begin{aligned}
\ell_{i, j}\left(\delta_{n} \cdot \bar{u}\right) & =\ell_{i, j}\left(\delta_{n}\right)+\ell_{\pi\left(\delta_{n}\right)^{-1}(i), \pi\left(\delta_{n}\right)^{-1}(j)}(\bar{u}) \\
& =\mathbf{1}_{i=1}+\ell_{[i-1]_{n},[j-1]_{n}}(\bar{u}),
\end{aligned}
$$

with the convention $\ell_{p, q}=\ell_{q, p}$ for $p>q$. Eventually we obtain

$$
\ell_{i, j}\left(\overline{\phi_{n}(u)}\right)=\ell_{[i-1]_{n},[j-1]_{n}}(\bar{u})+\mathbf{1}_{i=1}-\mathbf{1}_{[1+\pi(\bar{u})(n)]_{n}=j} .
$$


5.3. Action on templates. We now describe an action of a subgroup of bijections of $T_{n}$ on $T_{n}$ itself. Eventually, for any template $t \in T_{n}$, braids of $B_{n}\left(S_{n}, \ell, t\right)$ shall be in bijection with $B_{n}\left(S_{n}, \ell, t^{\prime}\right)$ whenever $t^{\prime}$ belongs in the orbit of $t$.

Definition 5.14. We define $G_{\Sigma_{n}}$, resp. $G_{\Sigma_{n}^{*}}$, to be the subgroup of bijections of $T_{n}$ generated by $\left\{\operatorname{inv}_{\Sigma_{n}}^{T}, \theta_{\Sigma_{n}}^{T}, \Phi_{n}^{T}\right\}$, resp. by $\left\{\operatorname{inv}_{\Sigma_{n}^{*}}^{T}, \phi_{n}^{T}\right\}$. For $t \in T_{n}$, we denote

$$
G_{\Sigma_{n}} \star t=\left\{g(t), g \in G_{\Sigma_{n}}\right\} \quad \text { and } \quad G_{\Sigma_{n}^{*}} \star t=\left\{g(t), g \in G_{\Sigma_{n}^{*}}\right\}
$$

the orbits of $t$ under the action of $G_{\Sigma_{n}}$, resp. $G_{\Sigma_{n}^{*}}$.

Lemma 5.15. For $n \geqslant 3$, we have $G_{\Sigma_{n}} \simeq(\mathbb{Z} / 2 \mathbb{Z})^{3}$ and $G_{\Sigma_{n}^{*}} \simeq \mathbb{Z} / 2 \mathbb{Z} \times \mathbb{Z} / n \mathbb{Z}$.

Proof. Let $u=\sigma_{i_{1}}^{e_{1}} \cdots \sigma_{i_{m}}^{e_{m}}$ be a $\Sigma_{n}$-word with $e_{1}, \ldots, e_{m} \in\{-1,+1\}$. From

$$
\begin{aligned}
& \operatorname{inv}_{\Sigma_{n}}\left(\theta_{\Sigma_{n}}(u)\right)=\operatorname{inv}_{\Sigma_{n}}\left(\sigma_{i_{1}}^{-e_{1}} \cdots \sigma_{i_{m}}^{-e_{m}}\right)=\sigma_{i_{m}}^{e_{m}} \cdots \sigma_{i_{1}}^{e_{1}}, \\
& \theta_{\Sigma_{n}}\left(\operatorname{inv}_{\Sigma_{n}}(u)\right)=\theta_{\Sigma_{n}}\left(\sigma_{i_{m}}^{-e_{m}} \cdots \sigma_{i_{1}}^{-e_{1}}\right)=\sigma_{i_{m}}^{e_{m}} \cdots \sigma_{i_{1}}^{e_{1}},
\end{aligned}
$$

we obtain that the maps $\operatorname{inv}_{\Sigma_{n}}$ and $\theta_{\Sigma_{n}}$ commute on $\Sigma_{n}$-words. Let $t$ be a template of $T_{n}$ and $v$ be a $\Sigma_{n}$-word representing a braid of template $t$. We have

$$
\left(\operatorname{inv}_{\Sigma_{n}}^{T} \circ \theta_{\Sigma_{n}}^{T}\right)(t)=\tau\left(\overline{\left(\operatorname{inv}_{\Sigma_{n}} \circ \theta_{\Sigma_{n}}\right)(v)}\right)=\tau\left(\overline{\left(\theta_{\Sigma_{n}} \circ \operatorname{inv}_{\Sigma_{n}}\right)(v)}\right)=\left(\theta_{\Sigma_{n}}^{T} \circ \operatorname{inv}_{\Sigma_{n}}^{T}\right)(t)
$$

and so $\operatorname{inv}_{\Sigma_{n}}^{T}$ and $\theta_{\Sigma_{n}}^{T}$ commute. Similar arguments establish the commutation of $\theta_{\Sigma_{n}}$ and $\Phi_{n}$, $\operatorname{inv}_{\Sigma_{n}}$ and $\Phi_{n}, \operatorname{inv}_{\Sigma_{n}^{*}}$ and $\phi_{n}$. We then obtain that $G_{\Sigma_{n}}$ and $G_{\Sigma_{n}^{*}}$ are quotient of

$$
H_{n}=\left\langle\operatorname{inv}_{\Sigma_{n}}^{T}\right\rangle \times\left\langle\theta_{\Sigma_{n}}^{T}\right\rangle \times\left\langle\Phi_{n}^{T}\right\rangle \quad \text { and } \quad H_{n}^{*}=\left\langle\operatorname{inv}_{\Sigma_{n}^{*}}^{T}\right\rangle \times\left\langle\phi_{n}^{T}\right\rangle .
$$

respectively. The maps $\theta_{\Sigma_{n}}^{T}$ and $\operatorname{inv}_{\Sigma_{n}}^{T}$ have order 2 since it is the case for $\theta_{\Sigma_{n}}$ and $\operatorname{inv}_{\Sigma_{n}}$ by construction. From Lemma 5.6 and Lemma 5.11 the map $\Phi_{n}$ and $\phi_{n}$ have order 2 and $n$ respectively. We then obtain the ismorphisms $H_{n} \simeq(\mathbb{Z} / 2 \mathbb{Z})^{3}$ and $H_{n}^{*} \simeq \mathbb{Z} / 2 \mathbb{Z} \times \mathbb{Z} / n \mathbb{Z}$.

Immediate computations establish that $G_{\Sigma_{n}} \star \tau\left(\sigma_{1} \sigma_{2}^{2}\right)$ and $G_{\Sigma_{n}^{*}} \star \tau\left(\sigma_{1}\right)$ have respectively 8 and $2 n$ elements and so we obtain

$$
G_{\Sigma_{n}}=H_{n} \simeq(\mathbb{Z} / 2 \mathbb{Z})^{3} \quad \text { and } \quad G_{\Sigma_{n}^{*}}=H_{n}^{*} \simeq \mathbb{Z} / 2 \mathbb{Z} \times \mathbb{Z} / n \mathbb{Z} .
$$

From a geometric point of view, the maps $\operatorname{inv}_{\Sigma_{n}}^{T}, \theta_{\Sigma_{n}}^{T}$ and $\Phi_{n}^{T} \circ \theta_{\Sigma_{n}}^{T}$ can be seen as reflections along the coordinate planes of the 3 -space and the result on $G_{\Sigma_{n}}$ is immediate. If we consider the base points of the braid evenly placed in a circumference, the maps $\operatorname{inv}_{\Sigma^{*}}^{T}$ and $\phi_{n}^{T}$ correspond respectively to a reflection along a plane and to a rotation of order $n$ along an axis orthogonal to this plane, establishing the result on $G_{\Sigma_{n}^{*}}$.

Remark. Note that for $n=2$, the map $\Phi_{n}$ and $\phi_{n}$ are trivial and that $\operatorname{inv}_{\Sigma_{n}}$ and $\theta_{\Sigma_{n}}$ are equals. Hence we obtain $G_{\Sigma_{n}} \simeq \mathbb{Z} / 2 \mathbb{Z} \simeq G_{\Sigma_{n}^{*}}$

Example 5.16. We recall that the template of a braid $\beta \in B_{4}$ is

$$
\tau(\beta)=\left(\pi(\beta), \ell_{1,2}(\beta), \ell_{1,3}(\beta), \ell_{2,3}(\beta), \ell_{1,4}(\beta), \ell_{2,4}(\beta), \ell_{3,4}(\beta)\right)
$$


The template of $\sigma_{1} \sigma_{2}^{-1}$ seen in $B_{4}$ is $t=((123), 1,-1,0,0,0,0)$. Using Propositions $5.3,5.4$ and 5.13 we obtain

$$
\begin{aligned}
\operatorname{inv}_{\Sigma_{4}}^{T}(t) & =((132), 0,-1,1,0,0,0), \\
\theta_{\Sigma_{4}}^{T}(t) & =((123),-1,1,0,0,0,0), \\
\Phi_{4}^{T}(t) & =((243), 0,0,0,0,-1,1), \\
\left(\operatorname{inv}_{\Sigma_{4}}^{T}(t) \circ \theta_{\Sigma_{4}}^{T}\right)(t) & =((132), 0,1,-1,0,0,0), \\
\left(\operatorname{inv}_{\Sigma_{4}}^{T} \circ \Phi_{4}^{T}\right)(t) & =((234), 0,0,1,0,-1,0), \\
\left(\theta_{\Sigma_{4}}^{T} \circ \Phi_{4}^{T}\right)(t) & =((243), 0,0,0,0,1,-1), \\
\left(\operatorname{inv}_{\Sigma_{4}}^{T} \circ \theta_{\Sigma_{4}}^{T} \circ \Phi_{4}^{T}\right)(t) & =((234), 0,0,-1,0,1,0),
\end{aligned}
$$

and so the set $G \star t$ has exactly 8 elements.

5.4. Template reduction. Now we define a total ordering on $T_{n}$. We start with permutations of $\mathfrak{S}_{n}$.

Definition 5.17. For $\sigma$ and $\sigma^{\prime}$ two permutations of $\mathfrak{S}_{n}$ we write $\sigma<\sigma^{\prime}$ whenever

$$
(\sigma(1), \ldots, \sigma(n))<_{\operatorname{CoLEx}}\left(\sigma^{\prime}(1), \ldots, \sigma^{\prime}(n)\right),
$$

i.e., whenever there exists $k \in[1, n]$ such that $\sigma(n)=\sigma^{\prime}(n), \ldots, \sigma(k+1)=\sigma^{\prime}(k+1)$ and $\sigma(k)<\sigma^{\prime}(k)$.

For example, the ordering of permutations occurring in Example 5.16 is

$$
(234)<(243)<(123)<(132) \text {. }
$$

Definition 5.18. For two templates $t=\left(\sigma,\left(\ell_{i, j}\right)_{1 \leqslant i<j \leqslant n}\right)$ and $t^{\prime}=\left(\sigma^{\prime},\left(\ell_{i, j}^{\prime}\right)_{1 \leqslant i<j \leqslant n}\right)$ we write $t<t^{\prime}$ whenever

$$
\left(\sigma, \ell_{1,2}, \ldots, \ell_{1, n}, \ldots, \ell_{n-1, n}\right)<_{\text {Lex }}\left(\sigma^{\prime}, \ell_{1,2}^{\prime}, \ldots, \ell_{1, n}^{\prime}, \ldots, \ell_{n-1, n}^{\prime}\right),
$$

where we recall the integers $\ell_{i, j}(\beta)$ are enumerated following a co-lexicographic ordering on their indices (see Definition 4.9). For a template $t$ we denote by $\operatorname{red}_{S_{n}}(t)$ the minimal element of $G_{S_{n}} \star t$. We say that a template $t \in T_{n}$ is $S_{n}$-reduced if $\operatorname{red}_{S_{n}}(t)=t$ holds. For an integer $\ell \in \mathbb{N}$ we denote by $R_{n}\left(S_{n}, \ell\right)$ the set of reduced templates lying in $T_{n}\left(S_{n}, \ell\right)$.

Example 5.19. We reconsider template $t$ of Example 5.16. By (23) we obtain

$$
\operatorname{red}_{\Sigma_{4}}(t)=((234), 0,0,-1,0,1,0) .
$$

which is equal to $\left(\operatorname{inv}_{\Sigma_{4}}^{T} \circ \theta_{\Sigma_{4}}^{T} \circ \Phi_{4}^{T}\right)(t)$.

Proposition 5.20. For $\mu$ a $S_{n}$-stable map of $S_{n}$-words, $\ell$ an integer $\geqslant 0$ and $t a$ template of $T_{n}$ we have

$$
\bar{\mu}\left(B_{n}\left(S_{n}, \ell, t\right)\right)=B_{n}\left(S_{n}, \ell, \mu^{T}(t)\right)
$$

and $\operatorname{card}\left(B_{n}\left(S_{n}, \ell, t\right)\right)=\operatorname{card}\left(B_{n}\left(S_{n}, \ell, \mu^{T}(t)\right)\right)$.

Proof. A direct consequense of Definition 5.1 and Lemma 5.2.

Corollary 5.21. Let $\ell$ be an integer. We have

$$
s\left(B_{n}, S_{n} ; \ell\right)=\sum_{t \in \mathrm{R}_{n}\left(S_{n}, \ell\right)} \operatorname{card}\left(B_{n}\left(S_{n}, \ell, t\right)\right) \cdot \operatorname{card}\left(G_{S_{n}} \star t\right) .
$$


Proof. We have

$$
B_{n}\left(S_{n}, \ell\right)=\bigsqcup_{t \in T_{n}\left(S_{n}, \ell\right)} B_{n}\left(S_{n}, \ell, t\right)=\bigsqcup_{t_{r} \in \mathrm{R}_{n}\left(S_{n}, \ell\right)} \bigsqcup_{t \in G_{S_{n}} \star t_{r}} B_{n}\left(S_{n}, \ell, t\right)
$$

Assume $t_{r}$ is a template of $\mathrm{R}_{n}\left(S_{n}, \ell\right)$ and $t$ lies in $G_{S_{n}} \star t_{r}$. Then there exists a $S_{n}$-stable bijection $\mu \in G_{S_{n}}$ satisfying $t=\mu^{T}\left(t_{r}\right)$. It follows from Proposition 5.20 that the set $B_{n}\left(S_{n}, \ell, t\right)$ has the same cardinality as $B_{n}\left(S_{n}, \ell, t_{r}\right)$. So we obtain

$$
\begin{aligned}
s\left(B_{n}, S_{n} ; \ell\right) & =\operatorname{card}\left(B_{n}\left(S_{n}, \ell\right)\right)=\sum_{t_{r} \in \mathrm{R}_{n}\left(S_{n}, \ell\right)} \sum_{t \in G\left(S_{n}\right) \star t_{r}} \operatorname{card}\left(B_{n}\left(S_{n}, \ell, t\right)\right) \\
& =\sum_{t_{r} \in \mathrm{R}_{n}\left(S_{n}, \ell\right)} \operatorname{card}\left(B_{n}\left(S_{n}, \ell, t_{r}\right)\right) \cdot \operatorname{card}\left(G_{S_{n}} \star t_{r}\right) .
\end{aligned}
$$

5.5. Algorithmic improvement. We now give an improvement of the algorithms of Section 4 using Corollary 5.21. From Corollary 5.21 we know how to obtain $s\left(B_{n}, S_{n} ; \ell\right)$ from an enumeration of braids associated to a reduced template. As in Section 4 we assume we have a function $\operatorname{LoAdRed}(n, \ell, t)$ loading from the storage memory a representative set of $B_{n}\left(S_{n}, \ell, t\right)$ where $t$ is a reduced template. We also assume we have a function $\operatorname{SAVERED}(W, n, \ell, t)$ which saves a representative set $W$ of $B_{n}\left(S_{n}, \ell, t\right)$ whenever $t$ is a reduced template.

Enumerating only braids with a reduced template reduces the requirements of storage space. But there is a little difficulty. The template $t_{x}$ used in the call of LOAD line 6 of Algorithm 2 - TEMPREPSET is not necessarily reduced. However, thanks to Proposition 5.20 we have

$$
B_{n}\left(S_{n}, \ell, t\right)=\bar{g}^{-1}\left(B_{n}\left(S_{n}, \ell, \operatorname{red}_{S_{n}}(t)\right)\right),
$$

where $g^{T}(t)=\operatorname{red}_{S_{n}}(t)$. Hence if $W_{r}$ is a representative set of $B_{n}\left(S_{n}, \ell, \operatorname{red}_{S_{n}}(t)\right)$ then $W=g^{-1}\left(W_{r}\right)$ is a representative set of $B_{n}\left(S_{n}, \ell, t\right)$. We then obtain Algorithm 4 - LOADFROMRED that can return any representative set of $B_{n}\left(S_{n}, \ell, t\right)$ from the storage of braids of $S_{n}$-length $\ell$ with a reduced template.

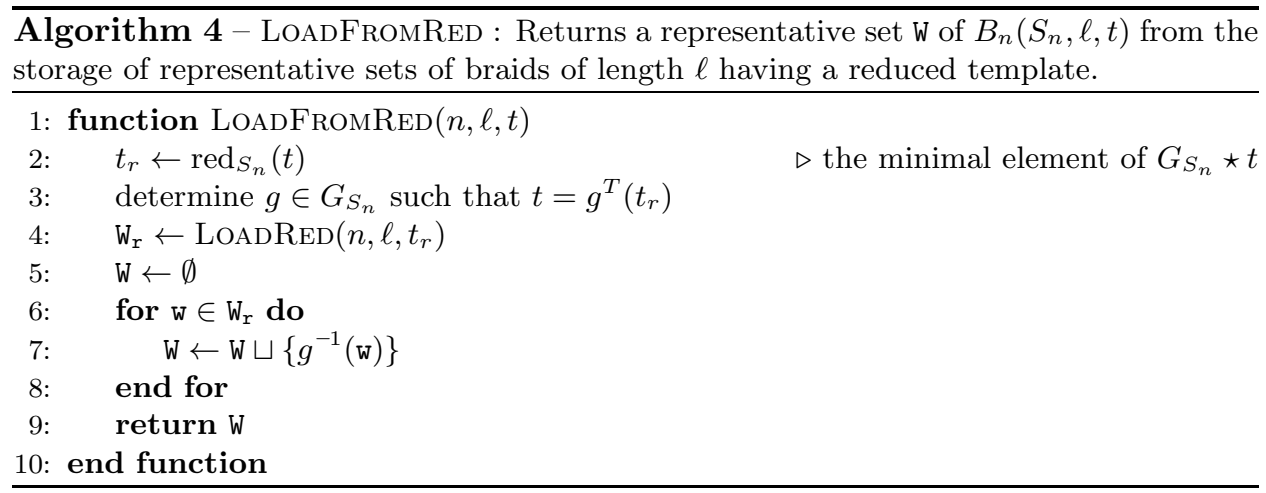

Replacing calls of LOAD by LOADRED and call of SAVE by SAVERED in Algorithm 2 - TempRepSet we obtain Algorithm RedTempRepSet $(\ell, t)$ which saves a representative set $\mathrm{W}_{\ell, t}$ of $B_{n}\left(S_{n}, \ell, t\right)$ and returns the pair $\left(\operatorname{card}\left(\mathrm{W}_{\ell}\right), \sum_{u \in \mathrm{W}_{\ell}} \omega_{S_{n}}(\bar{u})\right)$ for every integer $\ell \geqslant 1$ and every reduced template $t$ of $\mathrm{R}_{n}\left(S_{n}, \ell\right)$.

By Corollary 5.21 the number $s\left(B_{n}, S_{n} ; \ell\right)$ can be determined by running Algorithm REDTEMPREPSET on all reduced templates of $T_{n}\left(S_{n}, \ell\right)$. As for braids we 
can't determine reduced templates of $T_{n}\left(S_{n}, \ell\right)$ considering only reduced templates of $T_{n}\left(S_{n}, \ell-1\right)$. Assume we dispose of the set $\mathrm{R}_{n}\left(S_{n}, \ell-1\right)$ of reduced templates of $T_{n}\left(S_{n}, \ell-1\right)$. First we reconstruct the set $T_{n}\left(S_{n}, \ell-1\right)$ using

$$
T_{n}\left(S_{n}, \ell-1\right)=\left\{g(t) \text { for }(g, t) \in G_{S_{n}} \times \mathrm{R}_{n}\left(S_{n}, \ell-1\right)\right\} .
$$

As a second step we use (13) to obtain a supset $T_{\ell}^{\prime}$ of $T_{n}\left(S_{n}, \ell\right)$. Then we filter element of $T_{\ell}^{\prime}$ keeping only reduced templates by testing if a template is minimal in its orbit under the action of $G_{S_{n}}$. Eventually we obtain the set $\mathrm{R}_{n}^{\prime}\left(S_{n}, \ell\right)$ of reduced templates containing the reduced templates of $T_{n}\left(S_{n}, \ell\right)$. Moreover a template of $\mathrm{R}_{n}^{\prime}\left(S_{n}, \ell\right)$ is a reduced template of $T_{n}\left(S_{n}, \ell\right)$ if and only if there exists a braid $\beta$ of $B_{n}\left(S_{n}, \ell\right)$ having this precise template. We then obtain $\mathrm{R}_{n}\left(S_{n}, \ell\right)$ from the set $\mathrm{R}_{n}^{\prime}\left(S_{n}, \ell\right)$. These lead to Algorithm 5 - REDComBI, which is an improved version of Algorithm 3 - ComBI.

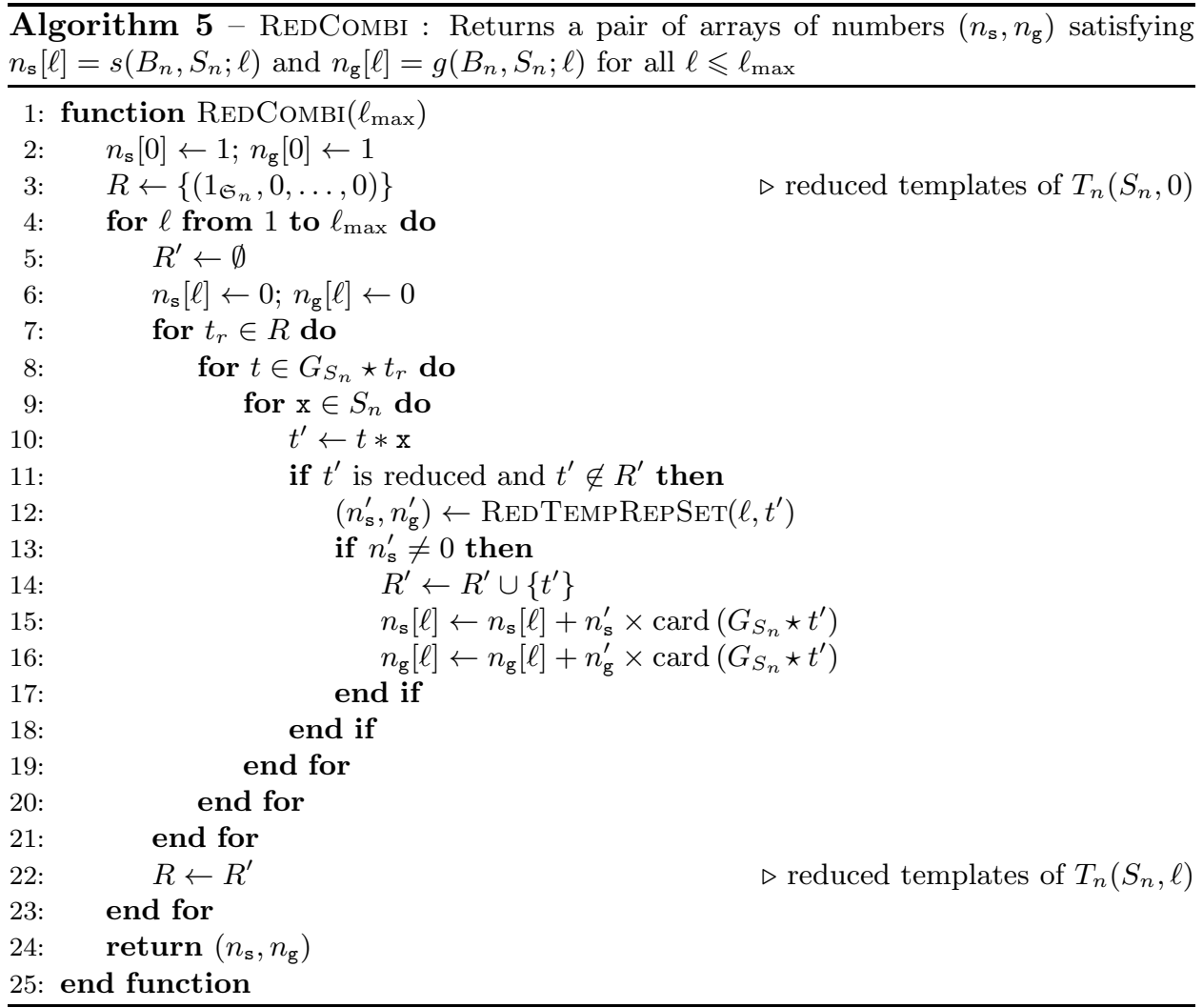

\section{Results}

For our experimentations we have coded a distributed version of Algorithm 5 REDCOMBI following a client / server model. Roughly speaking the server runs the core of Algorithm 5 while clients run Algorithm 4 - RedTempRepSet in parallel. Technical details are voluntarily omitted. The source code of our program is available on GitHub [20]. 
These programs were executed on a single computational node ${ }^{2}$ of the computing platform CALCULCO [25]. This node is equipped with 256 Go of RAM together with two processors AMD Epyc 7702 with 64 cores each for a total of 128 cores. In addition of this computational node we have used a distributed storage space of 30 To storing files containing representative sets.

6.1. Three strands. As values of $\mathcal{S}\left(B_{3}, \Sigma_{3}\right)$ and $\mathcal{G}\left(B_{3}, \Sigma_{3}\right)$ are already known since the work of L. Sabalka [24] we have started our experimentation on the dual presentation of $B_{3}$ (see Table 1$)$.

\begin{tabular}{r|r|r}
$\ell$ & $s\left(B_{3}, \Sigma_{3}^{*} ; \ell\right)$ & $g\left(B_{3}, \Sigma_{3}^{*} ; \ell\right)$ \\
\hline 0 & 1 & 1 \\
1 & 6 & 6 \\
2 & 20 & 30 \\
3 & 54 & 126 \\
4 & 134 & 498 \\
5 & 318 & 1926 \\
6 & 734 & 7410 \\
7 & 1662 & 28566 \\
8 & 3710 & 110658 \\
9 & 8190 & 431046 \\
10 & 17918 & 1687890
\end{tabular}

\begin{tabular}{r|r|r}
$\ell$ & $s\left(B_{3}, \Sigma_{3}^{*} ; \ell\right)$ & $g\left(B_{3}, \Sigma_{3}^{*} ; \ell\right)$ \\
\hline 11 & 38910 & 6639606 \\
12 & 83966 & 26216418 \\
13 & 180222 & 103827366 \\
14 & 385022 & 412169970 \\
15 & 819198 & 1639212246 \\
16 & 1736702 & 6528347778 \\
17 & 3670014 & 26027690886 \\
18 & 7733246 & 103853269650 \\
19 & 16252926 & 414639810486 \\
20 & 34078718 & 1656237864738 \\
21 & 71303166 & 6617984181606
\end{tabular}

TABle 1. Combinatorics of $B_{3}$ relatively to dual generators $\Sigma_{3}^{*}$.

Using Padé approximant on obtained values we can conjecture rational expression for the spherical and geodesic growth series of $B_{3}$ relatively to dual generators.

Conjecture 6.1. The spherical and geodesic growth series of $B_{3}$ relatively to dual generators are

$$
\mathcal{S}\left(B_{3}, \Sigma_{3}^{*}\right)=\frac{(t+1)\left(2 t^{2}-1\right)}{(t-1)(2 t-1)^{2}}, \quad \mathcal{G}\left(B_{3}, \Sigma_{3}^{*}\right)=\frac{12 t^{3}-2 t^{2}+3 t-1}{(2 t-1)(3 t-1)(4 t-1)}
$$

If the previous conjecture is true the growth rate of $s\left(B_{3}, \Sigma_{3}^{*} ; \ell\right)$ is 2 while that of $g\left(B_{3}, \Sigma_{3}^{*} ; \ell\right)$ is 4 .

6.2. Four strands. In her thesis [2], M. Albenque computes the value $s\left(B_{4}, \Sigma_{4} ; \ell\right)$ up to $\ell \leqslant 12$. Running our algorithm on the 128 -cores node of the CALCULCO platform we determine the spherical and geodesic combinatorics of $B_{4}$ relatively to Artin's generators up to length 25 (see Table 2). Unfortunately the obtained values do not allow us to guess a rational expression of $\mathcal{S}\left(B_{4}, \Sigma_{4}\right)$ or of $\mathcal{G}\left(B_{4}, \Sigma_{4}\right)$. For information the storage of all braids of $B_{4}$ with geodesic $\Sigma_{4}$-length $\leqslant 25$ and reduced templates requires 26 To of disk space.

In case of dual generators we have reached length 17 (see Table 3). Using Padé approximant on our values we can conjecture the value of the spherical growth series of $B_{4}$ relatively to dual generators.

Conjecture 6.2. The spherical growth series of $B_{4}$ relatively to dual generators is

$$
\mathcal{S}\left(B_{4}, \Sigma_{4}^{*}\right)=-\frac{(t+1)\left(10 t^{6}-10 t^{5}-3 t^{4}+11 t^{3}-4 t^{2}-3 t+1\right)}{(t-1)\left(5 t^{2}-5 t+1\right)\left(10 t^{4}-20 t^{3}+19 t^{2}-8 t+1\right)}
$$

\footnotetext{
${ }^{2}$ Financed by the project BQR CIMPA 2020 and the laboratory LMPA.
} 


\begin{tabular}{r|r|r}
$\ell$ & $s\left(B_{4}, \Sigma_{4} ; \ell\right)$ & $g\left(B_{4}, \Sigma_{4} ; \ell\right)$ \\
\hline 0 & 1 & 1 \\
1 & 6 & 6 \\
2 & 26 & 30 \\
3 & 98 & 142 \\
4 & 338 & 646 \\
5 & 1110 & 2870 \\
6 & 3542 & 12558 \\
7 & 11098 & 54026 \\
8 & 34362 & 229338 \\
9 & 105546 & 963570 \\
10 & 322400 & 4016674 \\
11 & 980904 & 16641454 \\
12 & 2975728 & 68614150
\end{tabular}

\begin{tabular}{r|r|r}
$\ell$ & $s\left(B_{4}, \Sigma_{4} ; \ell\right)$ & $g\left(B_{4}, \Sigma_{4} ; \ell\right)$ \\
\hline 13 & 9007466 & 281799158 \\
14 & 27218486 & 1153638466 \\
15 & 82133734 & 4710108514 \\
16 & 247557852 & 19186676438 \\
17 & 745421660 & 78004083510 \\
18 & 2242595598 & 316591341866 \\
19 & 6741618346 & 1283041428650 \\
20 & 20252254058 & 5193053664554 \\
21 & 60800088680 & 20994893965398 \\
22 & 182422321452 & 84795261908498 \\
23 & 547032036564 & 342173680884002 \\
24 & 1639548505920 & 1379691672165334 \\
25 & 4911638066620 & 5559241797216166
\end{tabular}

TABLE 2. Combinatorics of $B_{4}$ relatively to Artin's generators $\Sigma_{4}$.

\begin{tabular}{r|r|r}
$\ell$ & $s\left(B_{4}, \Sigma_{4}^{*} ; \ell\right)$ & $g\left(B_{4}, \Sigma_{4}^{*} ; \ell\right)$ \\
\hline 0 & 1 & 1 \\
1 & 12 & 12 \\
2 & 84 & 132 \\
3 & 478 & 1340 \\
4 & 2500 & 12788 \\
5 & 12612 & 117452 \\
6 & 62570 & 1053604 \\
7 & 303356 & 9311420 \\
8 & 1506212 & 81488628
\end{tabular}

\begin{tabular}{r|r|r}
$\ell$ & $S\left(B_{4}, \Sigma_{4}^{*} ; \ell\right)$ & $g\left(B_{4}, \Sigma_{4}^{*} ; \ell\right)$ \\
\hline 9 & 7348366 & 708368540 \\
10 & 35773324 & 6128211364 \\
11 & 173885572 & 52826999612 \\
12 & 844277874 & 454136092148 \\
13 & 4095929948 & 3895624824092 \\
14 & 19858981932 & 33359143410468 \\
15 & 96242356958 & 285259736104444 \\
16 & 466262144180 & 2436488694821748 \\
17 & 2258320991652 & 20790986096580060
\end{tabular}

TABle 3. Combinatorics of $B_{4}$ relatively to dual generators $\Sigma_{4}^{*}$.

If the previous conjecture is true, the growth rate of $s\left(B_{4}, \Sigma_{4}^{*} ; \ell\right)$ is given by the inverse of the maximal root of the denominator of (24), which is approximatively 4.8. Unfortunately we are not able to formulate such a conjecture for the geodesic growth series of $B_{4}$ relatively to dual generators.

Acknowledgments. The author wishes to thank the anonymous referee for his/her very sharp comments.

\section{REFERENCES}

[1] M. Albenque. Bijective combinatorics of positive braids. Electronic Notes in Discrete Mathematics, 29:225-229, 2007.

[2] M. Albenque. Tresses, animaux, cartes : à l'interaction entre combinatoire et probabilité. $\mathrm{PhD}$ thesis, Université Paris Diderot, 2008.

[3] M. Albenque and P. Nadeau. Growth function for a class of monoids. In 21st International Conference on Formal Power Series and Algebraic Combinatorics (FPSAC 2009), Discrete Math. Theor. Comput. Sci. Proc., AK, pages 25-38. Assoc. Discrete Math. Theor. Comput. Sci., Nancy, 2009.

[4] E. Artin. Theory of braids. Ann. of Math. (2), 48:101-126, 1947.

[5] D. Bessis. The dual braid monoid. Ann. Sci. École Norm. Sup. (4), 36(5):647-683, 2003.

[6] P. Biane and P. Dehornoy. Dual Garside structure of braids and free cumulants of products. Sém. Lothar. Combin., 72:Art. B72b, 15, 2014/15.

[7] J. Birman, K. H. Ko, and S. J. Lee. A new approach to the word and conjugacy problems in the braid groups. Adv. Math., 139(2):322-353, 1998. 
[8] A. Bronfman. Growth function of a class of monoids (preprint), 2001.

[9] R. Charney. Geodesic automation and growth functions for Artin groups of finite type. Math. Ann., 301(2):307-324, 1995.

[10] P. Dehornoy. Groupes de Garside. Ann. Sci. École Norm. Sup. (4), 35(2):267-306, 2002.

[11] P. Dehornoy. Combinatorics of normal sequences of braids. J. Combin. Theory Ser. A, 114(3):389-409, 2007.

[12] P. Dehornoy. Le calcul des tresses. Nano. Calvage \& Mounet, 2019.

[13] P. Dehornoy, F. Digne, E. Godelle, D. Krammer, and J. Michel. Foundations of Garside theory, volume 22 of EMS Tracts in Mathematics. European Mathematical Society (EMS), Zürich, 2015. Author name on title page: Daan Kramer.

[14] P. Dehornoy and L. Paris. Gaussian groups and Garside groups, two generalisations of Artin groups. Proc. London Math. Soc. (3), 79(3):569-604, 1999.

[15] Patrick Dehornoy, Ivan Dynnikov, Dale Rolfsen, and Bert Wiest. Ordering braids, volume 148 of Mathematical Surveys and Monographs. American Mathematical Society, Providence, RI, 2008.

[16] I. A. Dynnikov. On a Yang-Baxter mapping and the Dehornoy ordering. Uspekhi Mat. Nauk, 57(3(345)):151-152, 2002.

[17] D. B. A. Epstein, D. F. Holt, and S. E. Rees. The use of Knuth-Bendix methods to solve the word problem in automatic groups. volume 12, pages 397-414. 1991. Computational group theory, Part 2.

[18] R. Flores and J. González-Meneses. On the growth of Artin-Tits monoids and the partial theta function. arXiv:1808.03066, 2018

[19] L. Foissy and J. Fromentin. A divisibility result in combinatorics of generalized braids. $J$. Combin. Theory Ser. A, 152:190-224, 2017.

[20] J. Fromentin. Github - gbraids. https://github.com/jfromentin/gbraids, 2020.

[21] F. A. Garside. The braid group and other groups. Quart. J. Math. Oxford Ser. (2), 20:235254, 1969.

[22] F. Hivert, J.-C. Novelli, and J.-Y. Thibon. Sur une conjecture de Dehornoy. C. R. Math. Acad. Sci. Paris, 346(7-8):375-378, 2008.

[23] J. Mairesse and F. Mathéus. Growth series for Artin groups of dihedral type. Internat. J. Algebra Comput., 16(6):1087-1107, 2006.

[24] L. Sabalka. Geodesics in the braid group on three strands. In Group theory, statistics, and cryptography, volume 360 of Contemp. Math., pages 133-150. Amer. Math. Soc., Providence, RI, 2004.

[25] Calculco plateform. https://www-calculco.univ-littoral.fr, 2020.

[26] Wikipedia. Standard template librairy. https://en.wikipedia.org/wiki/Standard_ Template_Library, 2020.

Univ. Littoral Côte d'Opale, UR 2597, LMPA, Laboratoire de Mathématiques Pures et Appliquées Joseph Liouville, F-62100 Calais, France

Email address: fromentin@math.cnrs.fr 